\title{
Changing Views on Curves and Surfaces
}

\author{
Kathlén Kohn, Bernd Sturmfels and Matthew Trager
}

\begin{abstract}
Visual events in computer vision are studied from the perspective of algebraic geometry. Given a sufficiently general curve or surface in 3-space, we consider the image or contour curve that arises by projecting from a viewpoint. Qualitative changes in that curve occur when the viewpoint crosses the visual event surface. We examine the components of this ruled surface, and observe that these coincide with the iterated singular loci of the coisotropic hypersurfaces associated with the original curve or surface. We derive formulas, due to Salmon and Petitjean, for the degrees of these surfaces, and show how to compute exact representations for all visual event surfaces using algebraic methods.
\end{abstract}

\section{Introduction}

Consider a curve or surface in 3-space, and pretend you are taking a picture of that object with a camera. If the object is a curve, you see again a curve in the image plane. For a surface, you see a region bounded by a curve, which is called image contour or outline curve. The outline is the natural sketch one might use to depict the surface, and is the projection of the critical points where viewing lines are tangent to the surface. In both cases, the image curve has singularities that arise from the projection, even if the original curve or surface is smooth. Now, let your camera travel along a path in 3-space. This path naturally breaks up into segments according to how the picture looks like. Within each segment, the picture looks alike, meaning that the topology and singularities of the image curve do not change.

The appearance of a solid object under a continuously varying viewpoint was studied in the 1970s by Koenderink and van Doorn [20]. Their motivation came from visual perception in psychology and artificial intelligence. Koenderink offers a detailed discussion in his remarkable book on Solid Shape [19]. On the mathematical side, the topic was studied in singularity theory by Arnol'd and others [2, 18, 30]. In that setting, the transitions between locally stable views are the non-generic singularities from catastrophe theory. These catastrophes have been classified for projection-generic surfaces. The catalogue consists of the following six visual events. The first three names are due to René Thom [39]:

(L) Local events: lip, beak-to-beak, and swallowtail.

(M) Multi-local events: tangent crossing, cusp crossing, and triple point. 
In the 1980s, visual events became a research topic in computer vision [6, 24, 26, 32]. Chapter 13 in the textbook by Forsyth and Ponce [14] offers an introduction in that context. The motivation in computer vision was to give a description of all possible appearances of a solid object using a finite number of stable views, or aspects. The overall structure of aspects and events is encoded in the aspect graph, in which vertices correspond to aspects, and edges correspond to visual events between stable views. Although these ideas never found much practical use, several algorithms for computing aspect graphs of algebraic surfaces were proposed. Test implementations involved both numerical and symbolic methods. Ponce and Kriegman [32] and Rieger [34] studied the case of orthographic projections of parametric algebraic surfaces. Methods for implicit algebraic surfaces were introduced by Petitjean et al. 26] for orthographic projections, and by Rieger [35] for perspective projections. All examples shown in these articles are very special low-degree surfaces. We here revisit this literature, now 25 years old, and develop it further for today's applied algebraic geometry.

Our model for the object to be viewed is a smooth variety $X$ of dimension one or two in complex projective space $\mathbb{P}^{3}$. We assume that $X$ is defined over $\mathbb{R}$ and the real locus $X_{\mathbb{R}}$ is Zariski dense in $X$. Taking a picture of $X_{\mathbb{R}}$ is modeled by the linear projection $\pi: \mathbb{P}^{3} \rightarrow-\mathbb{P}^{2}$ with center $z$ (for Zentrum). This defines a curve $C_{z}(X)$ in the image plane $\mathbb{P}^{2}$. If $X$ is a curve, then $C_{z}(X)$ is the closure of the image of $X$ under $\pi$. If $X$ is a surface, then $C_{z}(X)$ is the branch locus of $\pi$ restricted to $X$. This is the closure of the set of points in $\mathbb{P}^{2}$ whose corresponding viewing lines are tangent to $X$. Even though $X$ is smooth, the curve $C_{z}(X)$ has many singular points. For a surface viewed from a general viewpoint $z$, the only singularities in the contour are nodes and cusps. For a space curve, the image curve has only nodes. As the center $z$ changes, the structure of its singularities is locally constant. At some point, a transition occurs, and the singularity structure changes. The visual event surface $\mathcal{V}(X)$ is the Zariski closure in $\mathbb{P}^{3}$ of the set of these transition points. This definition can be extended to singular curves and surfaces by excluding the role of singular points on $X$.

The visual event surface $\mathcal{V}(X)$ is usually reducible. If $X$ is a general curve, then $\mathcal{V}(X)$ has three irreducible components. If $X$ is a general surface, then $\mathcal{V}(X)$ has five irreducible components. These arise from the six events in (L) and (M) above. We shall explain the geometry of these irreducible components, and we discuss how to compute them in practice. An important caveat for applications is the distinction between real and complex points. Algebraic methods do not distinguish between them. They apply to any complex curve or surface $X$ in $\mathbb{P}^{3}$. For any particular $X$ that is defined over $\mathbb{R}$, it can happen that some visual events are not seen on its real points, i.e., they might live in the set $X \backslash X_{\mathbb{R}}$ of complex points.

The focus in this paper lies on curves and surfaces that are general in the sense of algebraic geometry. Thus, for a surface $X$ in $\mathbb{P}^{3}$ of degree $d$, we assume that its equation is general among homogeneous polynomials of degree $d$ in four variables. For a curve $X$ in $\mathbb{P}^{3}$ of degree $d$ and genus $g$, we assume that it is a general point in the Hilbert scheme of such curves.

We now briefly describe the organization and main results in this article. Section 2 is devoted to ruled surfaces in $\mathbb{P}^{3}$ and to its subclass of developable surfaces. We introduce effective representations of ruled surfaces, and we show how to compute with these. This is relevant because all visual event surfaces are ruled. Their irreducible components are the ruled surfaces in the bottom rows of Figures 1 and 2, These arise as iterated singular loci of 
Chow and Hurwitz threefolds in $\operatorname{Gr}\left(1, \mathbb{P}^{3}\right)$, and of dual varieties in $\left(\mathbb{P}^{3}\right)^{*}$.

In Section 3 we develop the geometry of visual event surfaces for curves in $\mathbb{P}^{3}$. The three irreducible components are the tangential surface, edge surface and trisecant surface. These represent the three Reidemeister moves on the image curve, as shown in Figure 3 . We present case studies that show the computation of visual event surfaces for curves up to degree six.

Section 4 concerns the visual event surface $\mathcal{V}(X)$ of a general surface $X$ in $\mathbb{P}^{3}$. The six events in (L) and (M) are depicted in Figure 4, which we discuss in detail. These events are translated into the algebraic setting, where they correspond to the five irreducible components of $\mathcal{V}(X)$. Their degrees are listed in Theorem 4.1. These formulas were known classically: they appear in paragraphs 597, 598, 599, 608 and 613 of Salmon's book [36]. Modern proofs were given by Petitjean [25]. In Section 5 we present new proofs, based on intersection theory in algebraic geometry, as seen in the textbook by Eisenbud and Harris [12].

Section 6 is devoted to practical methods for computing and representing the visual events associated with a surface $X$ in $\mathbb{P}^{3}$. This is a non-trivial matter because the degrees of the ruled surfaces in the output are very high, as seen in Table 2. For instance, if $X$ is a quintic, then the degrees of the irreducible components of $\mathcal{V}(X)$ range between 260 and 930.

\section{Ruled Surfaces and Developable Surfaces}

An irreducible surface in $\mathbb{P}^{3}$ is ruled if it is covered by straight lines. These lines are parameterized by some curve $C$, and they are known as the generators of the surface. A first example are smooth quadratic surfaces in $\mathbb{P}^{3}$. These possess two rulings of lines over $\mathbb{C}$. We refer to the book by Edge [11] for many classical results on ruled surfaces. In this section we develop algebraic tools for computing and representing ruled surfaces in practice.

Ruled surfaces arise naturally when taking pictures of an object in 3-space. We encounter them because all components of a visual event surface $\mathcal{V}(X)$ are ruled. Indeed, every general point $z$ on $\mathcal{V}(X)$ determines a line of sight that has a special intersection with the curve or surface $X$. Every point on the line shares this property with $z$ and hence lies in $\mathcal{V}(X)$.

A key player is the Grassmannian of lines in $\mathbb{P}^{3}$, here denoted $\operatorname{Gr}\left(1, \mathbb{P}^{3}\right)$. This is a 4 dimensional variety, embedded in $\mathbb{P}^{5}$ via Plücker coordinates. Every line is associated with a sextuple $\left(p_{12}: p_{13}: p_{14}: p_{23}: p_{24}: p_{34}\right)$ given by the $2 \times 2$-minors of a $2 \times 4$-matrix whose kernel is the line. The Plücker coordinates satisfy the quadratic Plücker relation

$$
p_{12} p_{34}-p_{13} p_{24}+p_{14} p_{23}=0 .
$$

The coordinate ring of $\operatorname{Gr}\left(1, \mathbb{P}^{3}\right)$ is the polynomial ring in the six unknowns $p_{i j}$ modulo the principal ideal generated by (1). We can also represent a line in $\mathbb{P}^{3}$ by its dual Plücker coordinates $\left(q_{12}: q_{13}: q_{14}: q_{23}: q_{24}: q_{34}\right)$. These are the $2 \times 2$-minors of a $2 \times 4$-matrix whose rows are points that span the line. Primal and dual Plücker coordinates are related by $q_{i j}=\sigma_{(i j k l)} p_{k l}$, where $i, j, k, l$ are distinct indices, and $\sigma_{(i j k l)}$ is the sign of the permutation $(i j k l)$. Every line satisfying $p_{34} \neq 0$ has the parametric representation

$$
z(t)=\left(-p_{34}: t p_{34}: p_{14}-t p_{24}: t p_{23}-p_{13}\right)
$$


Consider now an irreducible curve $C$ in $\operatorname{Gr}\left(1, \mathbb{P}^{3}\right)$ which has degree $d$ in $\mathbb{P}^{5}$. We write $I_{C}$ for its prime ideal in the coordinate ring. The union of all lines on $C$ is a ruled surface $\mathcal{S}_{C}$ in $\mathbb{P}^{3}$.

Lemma 2.1. The ruled surface $\mathcal{S}_{C}$ is irreducible and it has degree $d$ in $\mathbb{P}^{3}$. Conversely, every irreducible ruled surface in $\mathbb{P}^{3}$ arises in this way from some irreducible curve $C \subset \operatorname{Gr}\left(1, \mathbb{P}^{3}\right)$.

Proof. This is one of the basic facts derived in Edge's book [11, Chapter I, §26].

The defining polynomial of the surface $\mathcal{S}_{C} \subset \mathbb{P}^{3}$ can be computed from the equations

$$
\left(\begin{array}{cccc}
0 & p_{12} & p_{13} & p_{14} \\
-p_{12} & 0 & p_{23} & p_{24} \\
-p_{13} & -p_{23} & 0 & p_{34} \\
-p_{14} & -p_{24} & -p_{34} & 0
\end{array}\right) \cdot\left(\begin{array}{l}
x_{1} \\
x_{2} \\
x_{3} \\
x_{4}
\end{array}\right)=\left(\begin{array}{l}
0 \\
0 \\
0 \\
0
\end{array}\right)
$$

We add these four bilinear forms to the ideal $I_{C}$, and then we saturate with respect to the irrelevant ideal $\left\langle p_{12}, p_{13}, p_{14}, p_{23}, p_{24}, p_{34}\right\rangle$ of $\mathbb{P}^{5}$. The resulting ideal is prime, and it describes the incidence correspondence of points on lines that are in the curve $C$. Now, by eliminating the unknowns $p_{i j}$, we obtain a principal homogeneous prime ideal in $\mathbb{R}\left[x_{1}, x_{2}, x_{3}, x_{4}\right]$. The generator of this ideal is the polynomial of degree $d$ that defines the desired surface.

This computation can be reversed. Given a surface $\mathcal{S}$ in $\mathbb{P}^{3}$, we can compute the Fano scheme of all lines on $\mathcal{S}$. This lives in $\operatorname{Gr}\left(1, \mathbb{P}^{3}\right)$. Its ideal in $\mathbb{R}\left[p_{12}, p_{13}, p_{14}, p_{23}, p_{24}, p_{34}\right]$ is obtained by substituting (2) into the equation of $\mathcal{S}$, extracting the coefficients of the resulting polynomial in $t$, and saturating their ideal by $\left\langle p_{34}\right\rangle$. The Fano scheme is usually empty or consists of points. However, if it is a curve $C$, then the surface is ruled and $\mathcal{S}=\mathcal{S}_{C}$.

The dual projective space $\left(\mathbb{P}^{3}\right)^{*}$ consists of all planes in $\mathbb{P}^{3}$. Homogeneous coordinates on $\left(\mathbb{P}^{3}\right)^{*}$ are denoted $\left(y_{1}: y_{2}: y_{3}: y_{4}\right)$. This point $y$ represents the plane $\left\{x \in \mathbb{P}^{3}: \sum_{i=1}^{4} y_{i} x_{i}=\right.$ $0\}$. Given an arbitrary subvariety $X$ in $\mathbb{P}^{3}$, its dual $X^{\vee} \subset\left(\mathbb{P}^{3}\right)^{*}$ is the Zariski closure of the set of all planes $y$ that are tangent at a smooth point of $X$. Typically, the dual $\mathcal{S}^{\vee}$ of a surface of degree at least two will be a surface in $\left(\mathbb{P}^{3}\right)^{*}$. However, it can happen that $\mathcal{S}^{\vee}$ is a curve. In that case, $\mathcal{S}$ is a developable surface. Each developable surface $\mathcal{S}$ is encoded by its dual curve $\mathcal{S}^{\vee}$ since we can recover the surface by the biduality relation $\mathcal{S}=\left(\mathcal{S}^{\vee}\right)^{\vee}$.

Theorem 2.2. Every developable surface $\mathcal{S}$ is a ruled surface, i.e., it satisfies $\mathcal{S}=\mathcal{S}_{C}$ for some curve $C$ in $\operatorname{Gr}\left(1, \mathbb{P}^{3}\right)$. For a curve $C$ in $\operatorname{Gr}\left(1, \mathbb{P}^{3}\right)$, the corresponding ruled surface $\mathcal{S}_{C}$ is developable if and only if all tangent lines of $C$ in $\mathbb{P}^{5}$ are contained in the Plücker quadric.

Proof. For the first statement see [11, Chapter V, §344]. The second is [4, Prop. 12.4.1].

A developable surface $\mathcal{S}=\mathcal{S}_{C}$ that is not a cone has three distinct encodings as a curve. First, there is the curve $C$ in the Grassmannian $\operatorname{Gr}\left(1, \mathbb{P}^{3}\right)$. Second, there is the dual curve $\mathcal{S}^{\vee}$ in $\left(\mathbb{P}^{3}\right)^{*}$. We saw how to recover $\mathcal{S}$ from these encodings. Finally, there is the edge of regression $E(\mathcal{S})$ which lies on the surface $\mathcal{S}$ in $\mathbb{P}^{3}$. Points in $E(\mathcal{S})$ are planes in $\left(\mathbb{P}^{3}\right)^{*}$ that intersect the curve $\mathcal{S}^{\vee}$ with multiplicity three. The surface $\mathcal{S}$ is the tangential surface of $E(\mathcal{S})$, i.e., it is the union of lines that are tangent to $E(\mathcal{S})$ (see [29, page 111]). This also verifies that $\mathcal{S}$ is indeed a ruled surface. The curves $E(\mathcal{S})$ and $\mathcal{S}^{\vee}$ are also related by a 
biduality relation; namely, $\mathcal{S}^{\vee}$ is the set of planes in $\mathbb{P}^{3}$ that intersect $E(\mathcal{S})$ with multiplicity three [27, Thm. 5.1]. Moreover, the tangent lines of $E(\mathcal{S})$ and $\mathcal{S}^{\vee}$ are dual to each other. This situation degenerates when the surface $\mathcal{S}$ is a cone, which means that its dual $\mathcal{S}^{\vee}$ is a plane curve. In that special case, the edge of regression $E(\mathcal{S})$ is the vertex of the cone $\mathcal{S}$.

We illustrate the three curve encodings of a developable surface with a simple example.

Example 2.3. Let $\mathcal{S}$ be the surface of degree six in $\mathbb{P}^{3}$ that is defined by the polynomial

$$
f=16 x_{2}^{3} x_{3}^{3}-27 x_{1}^{2} x_{3}^{4}+6 x_{1} x_{2}^{2} x_{3}^{2} x_{4}-27 x_{2}^{4} x_{4}^{2}+48 x_{1}^{2} x_{2} x_{3} x_{4}^{2}-16 x_{1}^{3} x_{4}^{3} .
$$

This is the surface in [37, $\S 3$, eqn. (9)]. We verify that $\mathcal{S}$ is developable by computing the ideal of its dual variety $\mathcal{S}^{\vee} \subset\left(\mathbb{P}^{3}\right)^{*}$. This shows that $\mathcal{S}^{\vee}$ is a smooth rational quartic curve:

$$
\left\langle y_{2} y_{3}-4 y_{1} y_{4}, y_{3}^{3}+4 y_{2} y_{4}^{2}, y_{1} y_{3}^{2}+y_{2}^{2} y_{4}, y_{2}^{3}+4 y_{1}^{2} y_{3}\right\rangle .
$$

The curve $C$ in the Grassmannian $\operatorname{Gr}\left(1, \mathbb{P}^{3}\right)$ that encodes the ruling of $\mathcal{S}=\mathcal{S}_{C}$ has the ideal

$$
I_{C}=\left\langle 2 p_{14}-p_{23}, p_{24}^{2}+3 p_{13} p_{34}, p_{13} p_{24}-9 p_{12} p_{34}, p_{23}^{2}-16 p_{12} p_{34}, p_{13}^{2}+3 p_{12} p_{24}\right\rangle .
$$

This ideal defines the Fano scheme of $\mathcal{S}$ in $\mathbb{P}^{5}$. Finally, the edge of regression $E(\mathcal{S})$ is the rational quartic curve $\left\{\left(s^{4}: s^{3} t: s t^{3}: t^{4}\right)\right\}$ in $\mathbb{P}^{3}$. The ideal of this curve equals

$$
\left\langle x_{2} x_{3}-x_{1} x_{4}, x_{3}^{3}-x_{2} x_{4}^{2}, x_{1} x_{3}^{2}-x_{2}^{2} x_{4}, x_{2}^{3}-x_{1}^{2} x_{3}\right\rangle .
$$

This curve has $\mathcal{S}$ as its tangential surface. Note that (4) is isomorphic to (5). This reflects the isomorphism between (9) and (10) in [37]. All of these computations can be reversed. This example shows how various objects can serve as a representation of the surface $\mathcal{S}$.

Many of the ruled surfaces $\mathcal{S}_{C}$ we shall encounter in later sections have the property that their defining polynomial $f$ is extremely large and impossible to compute symbolically. In such cases, the curve $C$ in $\operatorname{Gr}\left(1, \mathbb{P}^{3}\right) \subset \mathbb{P}^{5}$ is more manageable, and we can often compute generators for its ideal $I_{C}$. This encoding of the ruling enables us to carry out computations with the surface $\mathcal{S}_{C}$. For example, suppose $\mathcal{S}_{C}$ has degree $d$ and consider a general line $L$ in $\mathbb{P}^{3}$. We may wish to compute the $d$ points in the intersection $\mathcal{S}_{C} \cap L$. This problem arises in our computer vision application when the camera travels along $L$. The real intersection points with the visual event surfaces are precisely the visual events we are interested in.

Fix two points $\left(a_{1}: a_{2}: a_{3}: a_{4}\right)$ and $\left(b_{1}: b_{2}: b_{3}: b_{4}\right)$ on $L$, and parameterize $L$ by

$$
x_{i}=s a_{i}+t b_{i} \quad \text { for } i=1,2,3,4 .
$$

To compute $\mathcal{S}_{C} \cap L$ from $I_{C}$, we substitute (6) into (3), we add the resulting four bilinear forms to $I_{C}$, we saturate with respect to $\left\langle p_{12}, \ldots, p_{34}\right\rangle$, and we then eliminate the six Plücker coordinates. The result is the principal ideal in $\mathbb{R}[s, t]$ that is generated by the binary form

$$
f\left(s a_{1}+t b_{1}, s a_{2}+t b_{2}, s a_{3}+t b_{3}, s a_{4}+t b_{4}\right) .
$$

Thus, even when $f$ is unknown, we can compute its specialization (7) directly from $I_{C}$. 
When $\mathcal{S}$ is developable, the specialization (7) can also be obtained from the ideal $I\left(\mathcal{S}^{\vee}\right)$. Let $J$ be a Jacobian matrix for the ideal $I\left(\mathcal{S}^{\vee}\right)$ in $\mathbb{R}\left[y_{1}, y_{2}, y_{3}, y_{4}\right]$. This matrix has four columns. Let $J_{x}$ be the matrix obtained from $J$ by adding one more row, namely the vector $\left(x_{1}, x_{2}, x_{3}, x_{4}\right)$ in (6). We now add the $3 \times 3$-minors of $J_{x}$ to the ideal $I\left(\mathcal{S}^{\vee}\right)$, we saturate with respect to the ideal of $2 \times 2$-minors of $J$, and then we eliminate the unknowns $y_{1}, y_{2}, y_{3}, y_{4}$. The result is the desired principal ideal (7) in $\mathbb{R}[s, t]$. See Example 6.6 for an application.

These strategies can be adapted to compute the plane curve that is obtained as the intersection of a ruled or developable surface $\mathcal{S}$ with a fixed plane $H$ in $\mathbb{P}^{3}$. For event surfaces, this corresponds to restricting the camera movement to a plane, or to assuming that all projections are orthographic (which means that the viewpoint lies on the plane at infinity). It is sufficient to parameterize the points on $H$ by writing $x_{i}=s a_{i}+t b_{i}+u c_{i}$ in (7).

Associated ruled surfaces. The ruled surfaces of interest to us arise from an arbitrary curve or surface $X$ in $\mathbb{P}^{3}$. They represent families of planes and lines that intersect $X$ with prescribed multiplicities and are shown in the bottom rows of Figures 1 and 2. For a general curve or surface $X$ in $\mathbb{P}^{3}$, the rows of these diagrams correspond to codimension in $\left(\mathbb{P}^{3}\right)^{*}$ or $\operatorname{Gr}\left(1, \mathbb{P}^{3}\right)$. The shown subvarieties consist of lines and planes that intersect $X$ with various multiplicities $m$. A solid edge from $Y_{1}$ to $Y_{2}$ means that $Y_{2}$ is an irreducible component of the singular locus of $Y_{1}$. A dashed edge just means that $Y_{2}$ is contained in $Y_{1}$. Below the ambient spaces $\left(\mathbb{P}^{3}\right)^{*}$ and $\operatorname{Gr}\left(1, \mathbb{P}^{3}\right)$ we see the coisotropic hypersurfaces studied by Gel'fand, Kapranov and Zelevinsky in [15, Sec. 4.3.B]. See also [21, 38]. These codimension one loci are:

- the dual surface $X^{\vee}$ in $\left(\mathbb{P}^{3}\right)^{*}$,

- the Chow threefold $\operatorname{Ch}(X)$ in $\operatorname{Gr}\left(1, \mathbb{P}^{3}\right)$, consisting of lines that meet the curve $X$,

- the Hurwitz threefold $\operatorname{Hur}(X)$ in $\operatorname{Gr}\left(1, \mathbb{P}^{3}\right)$, of lines that are tangent to the surface $X$.

Every irreducible hypersurface in $\operatorname{Gr}\left(1, \mathbb{P}^{3}\right)$ is defined by one equation in Plücker coordinates, which is unique up to scaling and modulo the Plücker quadric. In the two cases above, this equation is called Chow form resp. Hurwitz form of $X$. As we shall see in Sections 3 and 4. the irreducible components of the visual event surface of $X$ are (iterated) singular loci of the coisotropic hypersurfaces associated to $X$. The developable components are dual to the singular curves in the dual surface $X^{\vee}$. The non-developable components are parameterized by the singular curves in the singular locus of the Chow or Hurwitz threefold of $X$.

We first consider a general smooth curve $X$ in $\mathbb{P}^{3}$. The left diagram in Figure 1 depicts the landscape in $\left(\mathbb{P}^{3}\right)^{*}$. The dual surface $X^{\vee}$ consists of planes that meet $X$ with multiplicity 2. The singular locus of $X^{\vee}$ is the union of two irreducible curves, whose points are osculating planes $(m=3)$ and bitangent planes $(m=2+2)$. The symbols that denote our loci, like $\mathcal{T}^{p}(X)$ and $\mathcal{E}^{p}(X)$, will be explained in Sections 3 and 4 . The right diagram in Figure 1 shows the landscape in the Grassmannian $\operatorname{Gr}\left(1, \mathbb{P}^{3}\right)$. We refer to [22, Thm. 1.1 and Sec. 7] for precise statements and proofs, also for the right diagram in Figure 2, The singular locus of the Chow threefold $\operatorname{Ch}(X)$ is the surface $\operatorname{Sec}(X)$ in $\operatorname{Gr}\left(1, \mathbb{P}^{3}\right)$ of secant lines, i.e., lines that meet $X$ twice. The singular locus of $\operatorname{Sec}(X)$ is the curve $\mathcal{D}^{\ell}(X)$ of trisecant lines. The curve $\mathcal{T}^{\ell}(X)$ of tangent lines is contained in $\operatorname{Sec}(X)$ but it does not belong to the singular locus. 


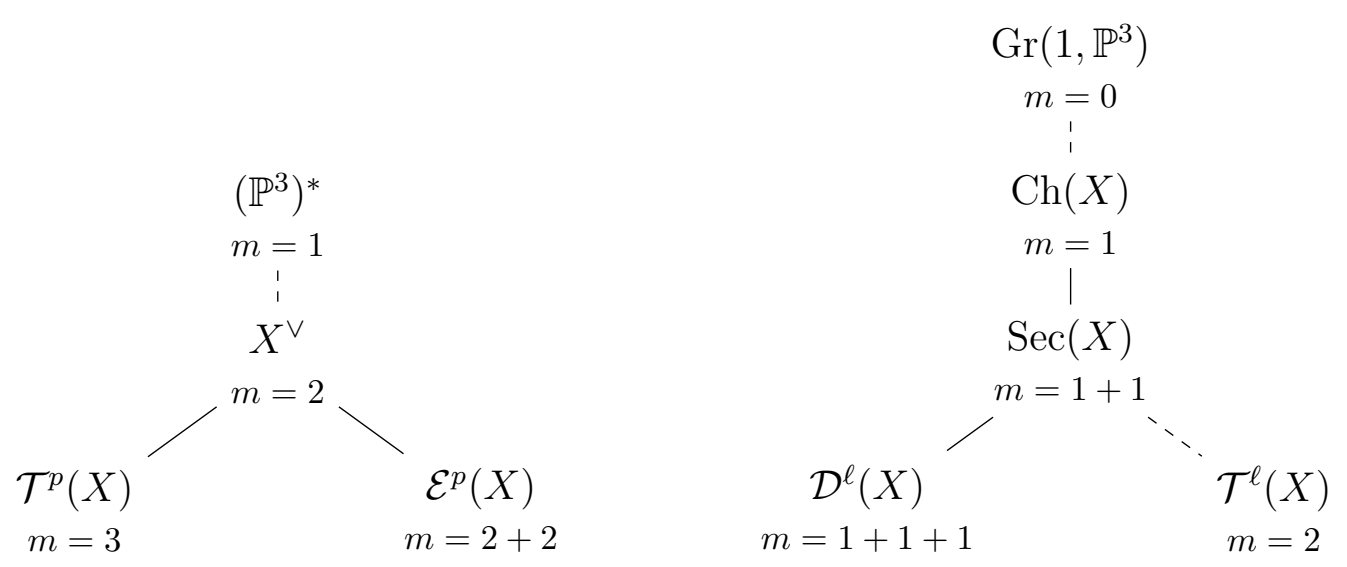

Figure 1: Loci of planes and lines that meet a curve $X$ with assigned multiplicities.

In Figure 2, we consider various loci associated with a general smooth surface $X$ in $\mathbb{P}^{3}$. The dual surface $X^{\vee}$ is singular along two irreducible curves. The nodal component $\mathcal{E}^{p}(X)$ of its singular locus is the set of all bitangent planes, and the cuspidal component $\mathcal{P}^{p}(X)$ is the set of all planes that intersect $X$ with multiplicity three at a point. The Hurwitz threefold $\operatorname{Hur}(X)$ is singular along two irreducible surfaces. Its nodal component $\operatorname{Bit}(X)$ contains all bitangent lines, and its cuspidal component $\mathrm{PT}(X)$ comprises all principal tangents, i.e., lines that meet $X$ with multiplicity three. The latter was denoted $\operatorname{Infl}(X)$ in [22]. These surfaces contain three special curves $\mathcal{F}^{\ell}(X), \mathcal{C}^{\ell}(X)$ and $\mathcal{T}^{\ell}(X)$, indicating lines that meet $X$ with multiplicity 4 , or $3+2$, or $2+2+2$. For instance, $2+2+2$ refers to tritangent lines. Sections 4, 5 and 6 are devoted to the ruled surfaces in $\mathbb{P}^{3}$ that are represented by these curves.

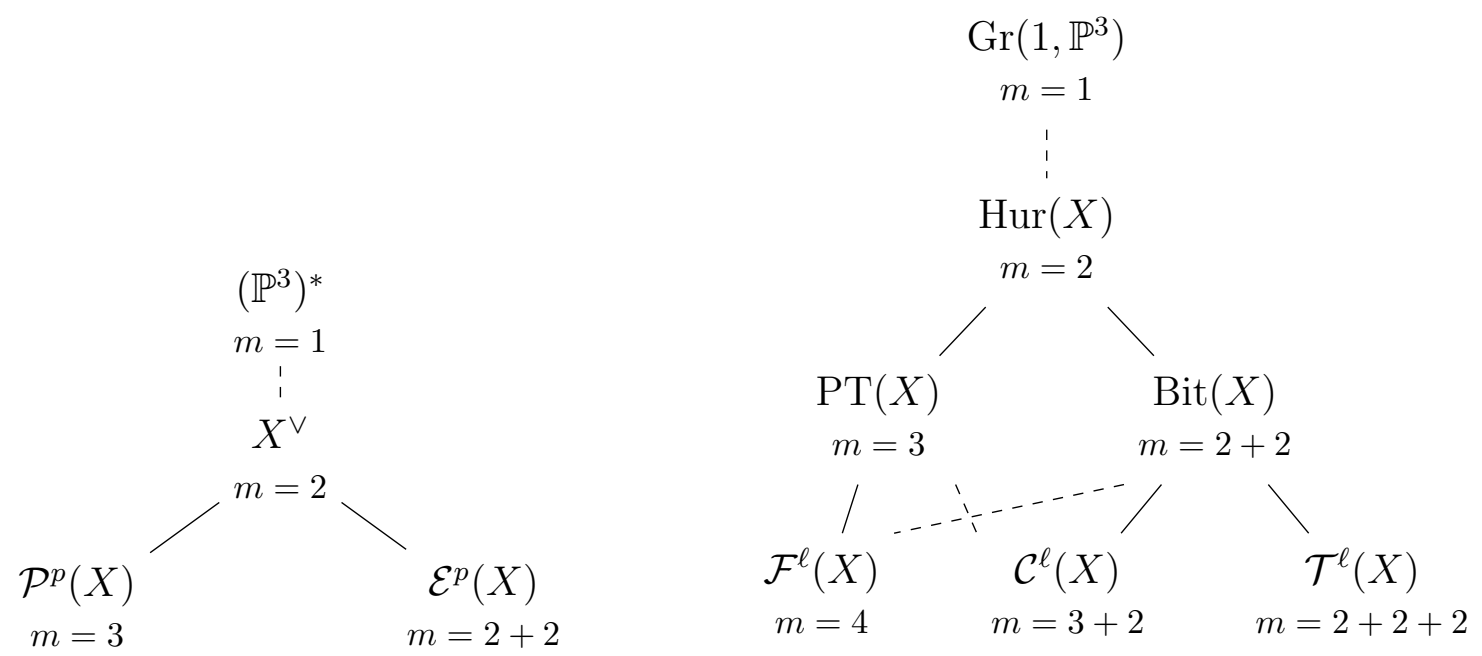

Figure 2: Loci of planes and lines that meet a surface $X$ with assigned multiplicities.

For each of the five curves at the bottom of Figure 2, there is also an associated curve on $X$. It consists of the points on $X$ where the special intersection occurs. For example, 
the curve associated with $\mathcal{E}^{p}(X)$ is the locus of points on $X$ that lie on bitangent planes. These are the contact points on a curved object when it is rolled on a table. Our favorite terminology for this curve is due to Cayley: he calls it the node-couple curve. For $\mathcal{P}^{p}(X)$ and $\mathcal{F}^{\ell}(X)$, the special contact occurs at a single point, and we can give a more detailed description. At a general point $x$, the surface has two principal tangents. These are the tangent lines of the nodal curve obtained by intersecting $X$ with its tangent plane at $x$. The same lines are the intersection of the tangent plane with the Hessian quadric at $x$ defined by

$$
y \cdot H_{f}(x) \cdot y^{T}=0, \quad \text { where } y=\left(y_{1}: y_{2}: y_{3}: y_{4}\right) \text { and } \quad H_{f}=\left(\frac{\partial^{2} f}{\partial x_{i} \partial x_{j}}\right) \text {. }
$$

Exceptional situations occur at flecnodal and parabolic points $x$. At a flecnodal point, one of the two principal tangents has intersection multiplicity four. Such a line is called a flecnodal line. At a parabolic point, the Hessian matrix $H_{f}(x)$ drops rank, and the two principal tangents degenerate to a double line. At these points, the intersection of $X$ with its tangent plane has a cusp at $x$. The locus of all parabolic points is the curve given by the intersection of $X$ with the Hessian surface $\left\{\operatorname{det}\left(H_{f}\right)=0\right\}$. Over the real numbers, the parabolic curve is the boundary between the elliptic and hyperbolic regions on $X$, where the two principal tangents are respectively both complex or both real.

The curve $\mathcal{P}^{p}(X)$ is the set of tangent planes at parabolic points, and the curve $\mathcal{F}^{\ell}(X)$ is the set of flecnodal lines at flecnodal points. The parabolic and flecnodal curves always intersect tangentially, at special points known as godrons (or cusps of the Gauss map). Interestingly, the node-couple curve also passes through the godrons, and has the same tangent as the parabolic and flecnodal curves [7, p. 170].

Remark 2.4. A formal study of the singular loci of the families of lines and planes described in this section presents many technical challenges. This is the topic of [22]. For example, in the course of examining parabolic surfaces, we discovered a small error in [3, Section 4], where Arrondo et al. consider the incidence variety for principal tangents of $X$ :

$$
Y_{2}=\left\{(x, L) \in X \times \operatorname{Gr}\left(1, \mathbb{P}^{3}\right): L \text { intersects } X \text { at } x \text { with multiplicity at least } 3\right\} .
$$

Lemma $4.1 \mathrm{~b})$ in [3] states that the surface $Y_{2}$ is singular at points $(x, L)$ for which $x$ is parabolic. This is incorrect. A general cubic surface $X$ has a degree 12 curve of parabolic points. However, the incidence variety $Y_{2}$ is smooth. This is shown by direct computation.

\section{Views of Curves}

In this section we study the visual events for a general curve $X$ in $\mathbb{P}^{3}$ of degree $d$ and genus $g$. In particular, $X$ is smooth and irreducible. The three visual events correspond to the three Reidemeister moves that are familiar from knot theory. They are shown in Figure 3.

The three components of the visual event surface of a space curve $X$ are as follows:

1. The tangential surface $\mathcal{T}(X)$, also known as the tangent developable, is the union of all tangent lines to $X$. It represents viewpoints $z$ such that the plane curve $C_{z}(X)$ has a cusp. When $z$ crosses $\mathcal{T}(X)$, a node on $C_{z}(X)$ transitions from being real to complex. 

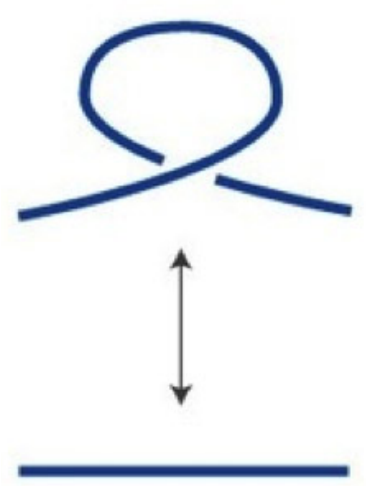
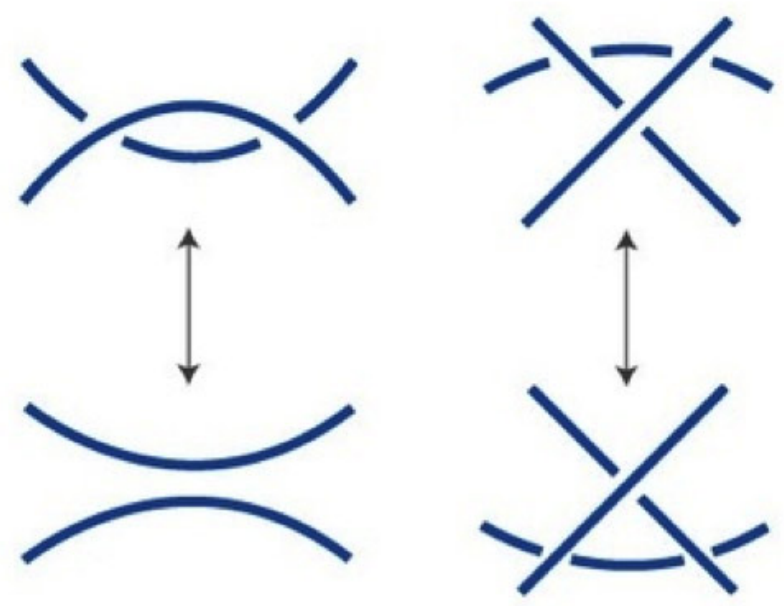

Figure 3: Changing views of a curve correspond to Reidemeister moves. The viewpoint $z$ crosses the tangential surface (left), edge surface (middle), or trisecant surface (right).

2. The edge surface $\mathcal{E}(X)$ is the union of all secant lines that are edges. An edge is the line spanned by two points on $X$ whose tangent lines lie in a common plane. This surface represents viewpoints $z$ such that the plane curve $C_{z}(X)$ has a tacnode. When $z$ crosses $\mathcal{E}(X)$, a pair of nodes transitions between being real and being complex.

3. The trisecant surface $\mathcal{D}(X)$ is the union of all lines that are spanned by triples of collinear points on $X$ (the symbol $\mathcal{D}$ stands for $d r e i$ ). This represents viewpoints $z$ such that $C_{z}(X)$ has a triple point. When $z$ crosses $\mathcal{D}(X)$, the real curve $C_{z}(X)$ experiences a triangle crossing, but the real singularity structure is unchanged.

The following classical theorem characterizes the expected degrees of these ruled surfaces.

Theorem 3.1. For a general space curve $X$ of degree $d$ and genus $g$, the degrees of the tangential surface $\mathcal{T}(X)$, the edge surface $\mathcal{E}(X)$ and the trisecant surface $\mathcal{D}(X)$ are as follows:

$$
\begin{array}{rcc}
\operatorname{deg}(\mathcal{T}(X)) & = & 2(d+g-1), \\
\operatorname{deg}(\mathcal{E}(X)) & = & 2(d-3)(d+g-1), \\
\operatorname{deg}(\mathcal{D}(X)) & = & \frac{(d-1)(d-2)(d-3)}{3}-(d-2) g .
\end{array}
$$

Proof. The degree of the tangential surface $\mathcal{T}(X)$ is the Riemann-Hurwitz number $2 d+2 g-2$. This coincides with the degree of the dual surface $X^{\vee}$. See [29, page 111] for a geometric derivation and [17] for computational examples. The formula for the degree of the edge surface $\mathcal{E}(X)$ appears in [33, Theorem 2.1]. The proof given there is based on De Jonquiéres' Formula. The degree of the trisecant surface $\mathcal{D}(X)$ is due to Berzolari who first found it in 1895. One finds Berzolari's formula in Bertin's article [5] on the geometry of $\mathcal{D}(X)$.

Table 1 summarizes the conclusion of Theorem 3.1 for space curves of degree $d$ at most six. The genus $g$ ranges from 0 to Castelnuovo's bound. Note that, for fixed $d$ and increasing 


\begin{tabular}{ccccc}
\hline$d$ & $g$ & $\operatorname{deg}(\mathcal{T}(X))$ & $\operatorname{deg}(\mathcal{E}(X))$ & $\operatorname{deg}(\mathcal{D}(X))$ \\
\hline 3 & 0 & 4 & 0 & 0 \\
4 & 0 & 6 & 6 & 2 \\
4 & 1 & 8 & 8 & 0 \\
5 & 0 & 8 & 16 & 8 \\
5 & 1 & 10 & 20 & 5 \\
5 & 2 & 12 & 24 & 2 \\
6 & 0 & 10 & 30 & 20 \\
6 & 1 & 12 & 36 & 16 \\
6 & 2 & 14 & 42 & 12 \\
6 & 3 & 16 & 48 & 8 \\
6 & 4 & 18 & 54 & 4 \\
\hline
\end{tabular}

Table 1: Degrees of the components of the visual event surface of a space curve

$g$, the degree of $\mathcal{D}(X)$ decreases while that of the others increases. In particular, there is no trisecant surface for twisted cubic curves and elliptic quartic curves (cf. [5, Proposition 1]).

The edge surface $\mathcal{E}(X)$ is of importance in convex geometry because the algebraic boundary of the convex hull of a real affine curve $X_{\mathbb{R}}$ consists of $\mathcal{E}(X)$ and the tritangent planes of $X$. This was shown by Ranestad and Sturmfels in [33, §2], and in [33, §3] they describe a method for computing $\mathcal{E}(X)$ when $X$ is rational. This theme was picked up by Seigal and Sturmfels in their study of real tensor decompositions [37]. According to [37, §3], the real rank two boundary of $X$ is the union $\mathcal{T}(X) \cup \mathcal{E}(X)$, so it is part of the visual event surface of $X$. The study of curves in the present section is thus a further development of [33, 37].

Consider now the projection $\pi_{z}: X \subset \mathbb{P}^{3} \rightarrow-\rightarrow \mathbb{P}^{2}$ from a center $z \in \mathbb{P}^{3} \backslash X$. The target $\mathbb{P}^{2}$ has two intrinsic realizations. These live in the ambient spaces $\operatorname{Gr}\left(1, \mathbb{P}^{3}\right)$ and $\left(\mathbb{P}^{3}\right)^{*}$ respectively. The first is the surface $\alpha(z)$ of all lines in $\mathbb{P}^{3}$ that contain $z$. The second is the plane $z^{\vee}$ of all planes in $\mathbb{P}^{3}$ that contain $z$. Basic projective geometry yields the following characterizations of the image curve $C_{z}(X)$ in these intrinsic realizations of the image plane.

Proposition 3.2. The image $C_{z}(X)$ of our curve $X$ is projectively equivalent to the curve $\alpha(z) \cap \mathrm{Ch}(X)$ in the Grassmannian $\operatorname{Gr}\left(1, \mathbb{P}^{3}\right)$. The dual curve $\left(C_{z}(X)\right)^{\vee}$ in $\left(\mathbb{P}^{2}\right)^{*}$ is projectively equivalent to the curve $z^{\vee} \cap X^{\vee}$ in the dual projective space $\left(\mathbb{P}^{3}\right)^{*}$.

In computer vision, the term visual cone is used for the union of all lines in the pre-image of a set in $\mathbb{P}^{2}$. The visual cone of a curve is a developable surface in $\mathbb{P}^{3}$. The dual of the visual cone associated with $C_{z}(X)$ is the plane curve $z^{\vee} \cap X^{\vee}$. Hence, Proposition 3.2 describes the curves in $\operatorname{Gr}\left(1, \mathbb{P}^{3}\right)$ and $\left(\mathbb{P}^{3}\right)^{*}$ that encode this visual cone, as discussed after Theorem 2.2.

Our task in this section is to solve the following computer algebra problem. Given a general space curve $X$, compute the ruled surfaces $\mathcal{T}(X), \mathcal{E}(X)$ and $\mathcal{D}(X)$. Here the input is either the ideal of $X$, or a parametric representation of $X$. The output is the defining polynomial $f$ of the surface $\mathcal{S}_{C}$ in $\mathbb{P}^{3}$. If the polynomial $f$ is too large, we compute the ideal of the curve $C$ in $\operatorname{Gr}\left(1, \mathbb{P}^{3}\right)$ or, when $\mathcal{S}_{C}$ is developable, the ideal of the dual $\mathcal{S}_{C}^{\vee}$ in $\left(\mathbb{P}^{3}\right)^{*}$. 
We use the symbols $\mathcal{T}^{\ell}(X), \mathcal{E}^{\ell}(X)$ and $\mathcal{D}^{\ell}(X)$ to denote the curves in the Grassmannian $\operatorname{Gr}\left(1, \mathbb{P}^{3}\right)$ that represent the surfaces $\mathcal{T}(X), \mathcal{E}(X)$ and $\mathcal{D}(X)$. The degrees of these curves in $\mathbb{P}^{5}$ are the numbers in Theorem 3.1. Two of the curves appear on the lower right in Figure 1 .

The surfaces $\mathcal{T}(X)$ and $\mathcal{E}(X)$ are developable, so they can also be represented by their dual curves in $\left(\mathbb{P}^{3}\right)^{*}$. We use the same notation as the left diagram in Figure 1:

$$
\mathcal{T}^{p}(X):=\mathcal{T}(X)^{\vee} \quad \text { and } \quad \mathcal{E}^{p}(X):=\mathcal{E}(X)^{\vee} .
$$

Here the index " $p$ " stands for planes. The earlier used upper index " $\ell$ " stands for lines. The trisecant surface $\mathcal{D}(X)$ is ruled but not developable, so it has no associated curve in $\left(\mathbb{P}^{3}\right)^{*}$.

Many space curves arising in applied contexts lie in the zero set of a quadratic polynomial. A generic curve has this property when its genus $g$ is maximal with respect to the Castelnuovo bound [16, Thm. 6.4, page 351]. We now focus on that special case. Later in this section we address our computational task for general curves that are not on a quadric.

Let $X$ be a general curve on a smooth quadric $Q$ in $\mathbb{P}^{3}$. Any line $L$ in $\mathbb{P}^{3}$ that intersects the quadric $Q$ in three points must lie on $Q$. Hence $L$ lies in one of the two rulings of $Q$.

Remark 3.3. If $d \geq 4$ and $(d, g) \neq(4,1)$ and $X$ lies on a quadric $Q$, then the trisecant surface $\mathcal{D}(X)$ coincides with the quadric surface $Q$, taken with an appropriate multiplicity.

To derive that multiplicity, and to set the stage for computing $\mathcal{T}(X)$ and $\mathcal{E}(X)$, we perform a linear change of coordinates in $\mathbb{P}^{3}$ so that the equation of $Q$ equals $x_{1} x_{4}=x_{2} x_{3}$. Thus we identify $Q$ with the Segre surface $\mathbb{P}^{1} \times \mathbb{P}^{1}$. We fix affine coordinates $((1: s),(1: t))$.

Corollary 3.4. If $X$ has bidegree $(a, b)$ on $Q=\mathbb{P}^{1} \times \mathbb{P}^{1} \subset \mathbb{P}^{3}$, then the degrees of the tangential surface $\mathcal{T}(X)$, the edge surface $\mathcal{E}(X)$ and the (non-reduced) trisecant surface $\mathcal{D}(X)$ are

$$
\begin{array}{rcc}
\operatorname{deg}(\mathcal{T}(X)) & = & 2 a b, \\
\operatorname{deg}(\mathcal{E}(X)) & = & 2 a b(a+b-3), \\
\operatorname{deg}(\mathcal{D}(X)) & = & 2\left(\left(\begin{array}{l}
a \\
3
\end{array}\right)+\left(\begin{array}{l}
b \\
3
\end{array}\right)\right) .
\end{array}
$$

Proof. The affine polynomial $f(s, t)$ that defines $X$ has degree $a$ in $s$ and degree $b$ in $t$. Our curve $X$ has degree $d=a+b$ and genus $g=(a-1)(b-1)$. Indeed, it is a basic fact from toric geometry that the genus $g$ is the number of interior lattice points of the Newton polygon, which is a rectangle of size $a \times b$. Moreover, a general curve $X$ in $\mathbb{P}^{3}$ of that degree and genus lies on a quadric, so we can apply Theorem 3.1. We substitute $d=a+b$ and $g=(a-1)(b-1)$ into the formulas given there. This yields the formulas in Corollary 3.4 .

We now see that the "appropriate multiplicity" in Remark 3.3 is $\left(\begin{array}{l}a \\ 3\end{array}\right)+\left(\begin{array}{l}b \\ 3\end{array}\right)$. The two summands correspond to the two rulings of $Q$. Each line in the first ruling meets $X$ in $a$ points, so it counts as a trisecant with multiplicity $\left(\begin{array}{l}a \\ 3\end{array}\right)$, and ditto with $b$ for the second ruling.

We shall present algorithms for computing $\mathcal{T}(X)$ and $\mathcal{E}(X)$ from the affine polynomial $f(s, t)$ that defines $X$ in $\mathbb{P}^{1} \times \mathbb{P}^{1}$. Recall that a change of coordinates is required in order to apply our method in situations when $X$ is given by its ideal in $\mathbb{R}\left[x_{1}, x_{2}, x_{3}, x_{4}\right]$. We illustrate this point, and later our algorithms, for the case when $a=3, b=2$ and hence $d=5, g=2$. 
Example 3.5. Let $X$ be the bicanonical embedding of a genus 2 curve. It has degree 5 in $\mathbb{P}^{3}$. The curve is arithmetically Cohen-Macaulay. Its ideal is given by the $2 \times 2$-minors of

$$
\left(\begin{array}{lll}
\ell_{11} & \ell_{12} & q_{1} \\
\ell_{21} & \ell_{22} & q_{2}
\end{array}\right)
$$

The $\ell_{i j}$ are linear forms and the $q_{i}$ are quadratic forms, found by computing syzygies of $X$. Assuming $\ell_{11}, \ell_{12}, \ell_{21}, \ell_{22}$ to be linearly independent, we change coordinates and write this as

$$
\left(\begin{array}{ccc}
x_{1} & x_{3} & q_{1}\left(x_{1}, x_{2}, x_{3}, x_{4}\right) \\
x_{2} & x_{4} & q_{2}\left(x_{1}, x_{3}\right)
\end{array}\right)=\left(\begin{array}{ccc}
1 & t & g_{1}(s, t) \\
s & s t & g_{2}(t)
\end{array}\right) .
$$

Here, the polynomial $g_{1}$ has bidegree $(2,2)$ in $(s, t)$, the polynomial $g_{2}$ has degree 2 in $t$, and we used column operations to eliminate $x_{2}$ and $x_{4}$ from $q_{2}$. One of the $2 \times 2$-minors of this $2 \times 3$-matrix is the equation of bidegree $(3,2)$ that defines our curve in the affine plane:

$$
f(s, t)=g_{2}(t)-s g_{1}(s, t) .
$$

Conversely, every polynomial of bidegree $(3,2)$ in $(s, t)$ has a matrix representation (8).

To compute the tangential surface $\mathcal{T}(X)$ from $f(s, t)$, we form the $3 \times 4$-matrix

$$
M=\left(\begin{array}{cccc}
1 & s & t & s t \\
0 & -f_{t} & f_{s} & s f_{s}-t f_{t} \\
x_{1} & x_{2} & x_{3} & x_{4}
\end{array}\right), \quad \text { where } f_{s}=\frac{\partial f}{\partial s} \text { and } f_{t}=\frac{\partial f}{\partial t}
$$

The first two rows of $M$ are linearly independent, and they span the tangent line at the point of $X$ corresponding to $(s, t)$. The second row is the image of the tangent direction $\left(-f_{t}, f_{s}\right)$ of the affine curve $\{f=0\}$ under the linear map given by the Jacobian of $\mathbb{C}^{2} \rightarrow \mathbb{C}^{3},(s, t) \mapsto$ $(s, t, s t)$. Another point $\left(x_{1}: x_{2}: x_{3}: x_{4}\right)$ lies on that tangent line in $\mathbb{P}^{3}$ precisely when $\operatorname{rank}(M)=2$. The following ideal is generated by five polynomials in $\mathbb{R}\left[s, t, x_{1}, x_{2}, x_{3}, x_{4}\right]$ :

$$
\langle f\rangle+\langle 3 \times 3 \text {-minors of } M\rangle \text {. }
$$

Our argument implies the following method for finding the tangential surface of degree $2 a b$ :

Proposition 3.6. Eliminating the unknowns $s$ and $t$ from the ideal (9) yields a principal ideal in $\mathbb{R}\left[x_{1}, x_{2}, x_{3}, x_{4}\right]$. Its generator is the polynomial defining the tangential surface $\mathcal{T}(X)$.

Example 3.7. Let $d=5, g=2$ as in Example 3.5 , and fix the curve $X \subset \mathbb{P}^{1} \times \mathbb{P}^{1}$ defined by

$$
f=s^{3} t^{2}+s^{3}+t^{2}+s+t+1
$$

The polynomial that defines the tangential surface $\mathcal{T}(X)$ has degree 12 . It looks like this:

$93 x_{1}^{12}+310 x_{1}^{11} x_{2}+341 x_{1}^{10} x_{2}^{2}+558 x_{1}^{9} x_{2}^{3}+1054 x_{1}^{8} x_{2}^{4}+744 x_{1}^{7} x_{2}^{5}+837 x_{1}^{6} x_{2}^{6}+\cdots+216 x_{2} x_{4}^{11}+108 x_{4}^{12}$.

This polynomial has 432 monomials, out of the $\left(\begin{array}{c}15 \\ 3\end{array}\right)=455$ possible ones of degree 12 . 
We now consider the edge surface $\mathcal{E}(X)$. Its degree is $2 a b(a+b-3)$. We shall compute its dual representation $\mathcal{E}^{p}(X)$. Planes in $\mathbb{P}^{3}$ correspond to curves of bidegree $(1,1)$ in $\mathbb{P}^{1} \times \mathbb{P}^{1}$ :

$$
y_{1}+y_{2} s+y_{3} t+y_{4} s t=0 .
$$

We solve for $s$, substitute into $f(s, t)$, and clear denominators. The result is a polynomial in one variable $t$ of degree $a+b$. We seek the condition that this has two double roots, corresponding to $m=2+2$ in Figure 1 . This condition defines the curve $\mathcal{E}^{p}(X)$ in $\left(\mathbb{P}^{3}\right)^{*}$.

Example 3.8. Let $d=5, g=2$ as before in Example 3.5. Fix the curve $X$ in Example 3.7 . We shall compute the dual curve $\mathcal{E}^{p}(X)$ to the edge surface. The moving curve (11) has five intersection points with the fixed curve $f=0$ in (10). Their $t$-coordinates are the roots of

$$
c_{0}+c_{1} t+c_{2} t^{2}+c_{3} t^{3}+c_{4} t^{4}+c_{5} t^{5}=0,
$$

where the $c_{i}$ are quadratic polynomials in $y_{1}, y_{2}, y_{3}, y_{4}$. Regarding the coefficients as unknowns, we pre-compute the ideal $\Delta_{(2,2)}(5) \subset \mathbb{R}\left[c_{0}, c_{1}, c_{2}, c_{3}, c_{4}, c_{5}\right]$ whose variety consists of quintics (12) with two double roots. The ideal $\Delta_{(2,2)}(5)$ has codimension 2 and degree 12. It is generated by 10 quintics in the $c_{i}$, as seen in the row labeled $\lambda=221$ in [23, Table 1]. Let $I$ be the ideal obtained from $\Delta_{(2,2)}(5)$ by replacing the $c_{i}$ with the quadrics in $y_{1}, y_{2}, y_{3}, y_{4}$ that represent the specific curve $X$, and then saturating by the irrelevant ideal $\left\langle y_{1}, y_{2}, y_{3}, y_{4}\right\rangle$. The variety of $I$ is the curve $\mathcal{E}^{p}(X)$ in $\left(\mathbb{P}^{3}\right)^{*}$. The ideal $I$ has 14 minimal generators, all of degree 10, with large integer coefficients. This is the dual representation of the edge surface.

Computing $\mathcal{E}(X)$ by directly dualizing $\mathcal{E}^{p}(X)$ rarely terminates in practice. It is easier to intersect $\mathcal{E}(X)$ with lines or planes, as explained in Section 2, around (6) and (7).

We now consider curves $X$ that need not lie on a quadric $Q$. Let us first assume that $X$ is the image of a variety $Y$ in a higher-dimensional space $\mathbb{P}^{d}$ under a linear projection $\alpha: \mathbb{P}^{d} \rightarrow \mathbb{P}^{3}$. This allows us to preprocess $Y$, especially if the Chow form of $Y$ is known.

This approach works well when $X$ is rational. Here $Y$ is the rational normal curve in $\mathbb{P}^{d}$, which is parameterized by $\left(1: t: t^{2}: \cdots: t^{d}\right)$. Let $\alpha: \mathbb{P}^{d} \rightarrow \mathbb{P}^{3}$ be the linear projection that maps $Y$ onto our curve $X$. We write $A$ for the $4 \times(d+1)$ matrix that represents $\alpha$.

We first explain the computation of the tangential surface $\mathcal{T}(X)$. Let $s$ be an unknown and let $Q$ be the skew-symmetric $4 \times 4$-matrix obtained from $(3)$ by substituting to dual Plücker coordinates. We form the ideal in $\mathbb{R}\left[s, q_{12}, q_{13}, \ldots, q_{34}\right]$ that is generated by the Plücker quadric $q_{12} q_{34}-q_{13} q_{24}+q_{14} q_{23}$ and the eight entries of the $4 \times 2$ matrix

$$
Q \cdot A \cdot\left(\begin{array}{ccccccc}
1 & s & s^{2} & s^{3} & s^{4} & \cdots & s^{d} \\
0 & 1 & 2 s & 3 s^{2} & 4 s^{3} & \cdots & d s^{d-1}
\end{array}\right)^{T} .
$$

Eliminating $s$ and saturating with respect to the $q_{i j}$ now yields the ideal of the curve $\mathcal{T}^{\ell}(X)$. From this we can compute the defining polynomial of $\mathcal{T}(X)$ via (3). The edge surface $\mathcal{E}(X)$ can be computed similarly. This was also discussed in [33, §2] and in [37, §3].

We now compute the trisecant surface $\mathcal{D}(X)$ of a rational curve $X$ of degree $d$ in $\mathbb{P}^{3}$. The Chow form of $Y$ is the resultant of two binary forms of degree $d$. We write this as the 
determinant of the Bézout matrix $B(r)$. This is a symmetric $d \times d$-matrix whose entries are linear forms in the Plücker coordinates $r_{i j}$ of $(d-2)$-planes in $\mathbb{P}^{d}$. For the formula we refer to equation (1.18) on page 402 in Section III.12.1 of [15]. The Bézout matrix for $d=6$ equals

$$
B(r)=\left(\begin{array}{ccccccc}
r_{12} & r_{13} & r_{14} & r_{15} & r_{16} & r_{17} \\
r_{13} & r_{14}+r_{23} & r_{15}+r_{24} & r_{16}+r_{25} & r_{17}+r_{26} & r_{27} \\
r_{14} & r_{15}+r_{24} & r_{16}+r_{25}+r_{34} & r_{17}+r_{26}+r_{35} & r_{27}+r_{36} & r_{37} \\
r_{15} & r_{16}+r_{25} & r_{17}+r_{26}+r_{35} & r_{27}+r_{36}+r_{45} & r_{37}+r_{46} & r_{47} \\
r_{16} & r_{17}+r_{26} & r_{27}+r_{36} & r_{37}+r_{46} & r_{47}+r_{56} & r_{57} \\
r_{17} & r_{27} & r_{37} & r_{47} & r_{57} & r_{67}
\end{array}\right)
$$

We shall use the following fact that is well-known in computer algebra; see [1, page 1228].

Lemma 3.9. The minors of the Bézout matrix $B(r)$ having size $d-k+1$ define an irreducible variety of codimension $k$ in the Grassmannian of $(d-2)$-planes in $\mathbb{P}^{d}$. General points $q$ on this variety represent pairs of univariate polynomials of degree $d$ that have $k$ common zeros.

The second exterior power $\wedge_{2} A$ of the matrix $A$ is a matrix of format $6 \times\left(\begin{array}{c}d+1 \\ 2\end{array}\right)$. We write $p=\left(p_{12}, p_{13}, p_{14}, p_{23}, p_{24}, p_{34}\right)$ for the Plücker coordinates of a line in $\mathbb{P}^{3}$. The preimage of the line $p$ under the projection $\alpha$ is the $(d-2)$-plane in $\mathbb{P}^{d}$ with Plücker coordinates $r=p \cdot \wedge_{2} A$.

Proposition 3.10. If the $4 \times(d+1)$-matrix $A$ is sufficiently general, then the ideal of $(d-2) \times$ $(d-2)$-minors of the matrix $B\left(p \cdot \wedge_{2} A\right)$ defines the curve $\mathcal{D}^{\ell}(X)$ of degree $2\left(\begin{array}{c}d-1 \\ 3\end{array}\right)$ in $\mathbb{P}^{5}$.

Proof. We use Lemma 3.9. Intersecting the curve $X$ with two planes in $\mathbb{P}^{3}$ amounts to solving two univariate polynomials of degree $d$. The Chow form of $X$ is the determinant of the specialized Bézout matrix $B\left(p \cdot \wedge_{2} A\right)$. The corank of that matrix is the number of common zeros. That number is 3 when the intersection of the two planes is a trisecant line of $X$. Thus, the line $p$ is in the trisecant curve $\mathcal{D}^{\ell}(X)$ precisely when $B\left(p \cdot \wedge_{2} A\right)$ has rank $\leq d-3$. By Lemma 2.1 and setting $g=0$ in Theorem 3.1. the degree of $\mathcal{D}^{\ell}(X)$ is $2\left(\begin{array}{c}d-1 \\ 3\end{array}\right)$.

For an illustration consider rational curves $X$ of degree $d=6$. Then $A$ is a $4 \times 7$ matrix, and $\wedge_{2} A$ is a $6 \times 21$ matrix. The ideal of $4 \times 4$-minors of the $6 \times 6$-matrix $B\left(p \cdot \wedge_{2} A\right)$ is minimally generated, modulo the Plücker relation, by 45 quartics. After saturating with respect to the irrelevant maximal ideal $\left\langle p_{12}, p_{13}, p_{14}, p_{23}, p_{24}, p_{34}\right\rangle$, we obtain the prime ideal $I_{C}$ of the curve $C$ of trisecants. The ideal $I_{C}$ has degree 20 and is generated by 10 cubics.

The success of this computation relied on writing the Chow form of $X$ as a determinant of a matrix whose entries are linear in Plücker coordinates. Eisenbud, Schreyer and Weyman [13, §4] proved that such a formula exists for all curves. See [13, Proposition 4.2] for a derivation of the Bézout matrix $B(r)$ from the perspective of Ulrich sheaves, and [13, Example 4.6] for an extension to hyperelliptic curves. Whenever we have such matrices explicitly, we get the surface $\mathcal{D}(X)$ by imposing the corank 3 constraints. Such matrix formulas for Chow forms can also be derived for curves $X$ in $\mathbb{P}^{3}$ that arise by intersecting certain nice varieties. 
Example 3.11. Let $X$ be the curve in $\mathbb{P}^{3}$ defined by the $3 \times 3$-minors of the $3 \times 4$-matrix

$$
M(x)=\left(\begin{array}{cccc}
x_{1}+x_{4} & x_{2}-x_{1} & x_{3}-x_{2} & x_{3}+x_{4} \\
x_{4}-x_{3} & x_{1}+x_{4} & x_{2}-x_{1} & x_{2}+x_{3} \\
x_{3}-x_{2} & x_{2}-x_{3}+x_{4} & x_{1}+x_{4} & x_{1}
\end{array}\right) .
$$

This curve has $(d, g)=(6,3)$. By computing syzygies, we can represent every curve of degree 6 and genus 3 via such a matrix with linear entries. This follows from the HilbertBurch Theorem. Let $Y$ be the variety of $3 \times 4$-matrices of rank $\leq 2$. The Chow form of $Y$ is the determinant of the following $6 \times 6$-matrix in dual Plücker coordinates for lines in $\mathbb{P}^{11}$ :

$$
\left(\begin{array}{cccccc}
r_{11,12} & r_{11,22}+r_{21,12} & r_{11,32}+r_{31,12} & r_{21,22} & r_{21,32}+r_{31,22} & r_{31,32} \\
r_{11,13} & r_{11,23}+r_{21,13} & r_{11,33}+r_{31,13} & r_{21,23} & r_{21,33}+r_{31,23} & r_{31,33} \\
r_{11,14} & r_{11,24}+r_{21,14} & r_{11,34}+r_{31,14} & r_{21,24} & r_{21,34}+r_{31,24} & r_{31,34} \\
r_{12,13} & r_{12,23}+r_{22,13} & r_{12,33}+r_{32,13} & r_{22,23} & r_{22,33}+r_{32,23} & r_{32,33} \\
r_{12,14} & r_{12,24}+r_{22,14} & r_{12,34}+r_{32,14} & r_{22,24} & r_{22,34}+r_{32,24} & r_{32,34} \\
r_{13,14} & r_{13,24}+r_{23,14} & r_{13,34}+r_{33,14} & r_{23,24} & r_{23,34}+r_{33,24} & r_{33,34}
\end{array}\right) .
$$

This matrix appears in [15, page 472]. We now replace the Plücker coordinates $r_{i j, k l}$ by linear forms in the six coordinates $q_{i j}=a_{i} b_{j}-b_{i} a_{j}$ for lines in $\mathbb{P}^{3}$. For instance, $r_{11,12}=q_{12}+q_{14}-q_{24}$, $r_{11,13}=-q_{12}+q_{13}+q_{24}-q_{34}, r_{11,14}=q_{13}+q_{14}-q_{34}, \ldots$ These linear forms are obtained by setting $r_{i j, k l}=M(a)_{i j} M(b)_{k l}-M(b)_{i j} M(a)_{k l}$ where $a=\left(a_{1}, a_{2}, a_{3}, a_{4}\right)$ and $b=\left(b_{1}, b_{2}, b_{3}, b_{4}\right)$.

The trisecant curve $\mathcal{D}^{\ell}(X)$ is defined in $\operatorname{Gr}\left(1, \mathbb{P}^{3}\right) \subset \mathbb{P}^{5}$ by the $4 \times 4$-minors of the resulting matrix 13. Saturating by $\left\langle q_{12}, q_{13}, q_{14}, q_{23}, q_{24}, q_{34}\right\rangle$ yields the prime ideal of $\mathcal{D}^{\ell}(X)$ :

$$
\begin{aligned}
I= & \left\langle q_{14} q_{23}-q_{13} q_{24}+q_{12} q_{34}, q_{13} q_{23}-q_{23}^{2}-q_{14} q_{24}-q_{24}^{2}+q_{12} q_{34}-2 q_{13} q_{34}+2 q_{14} q_{34}+2 q_{23} q_{34},\right. \\
& q_{12} q_{23}-q_{23}^{2}-q_{12} q_{24}+q_{13} q_{24}-3 q_{14} q_{24}-q_{23} q_{24}-q_{12} q_{34}+2 q_{14} q_{34}+3 q_{23} q_{34}+q_{24} q_{34}-2 q_{34}^{2}, \\
& q_{14}^{2}+q_{14} q_{24}+q_{24}^{2}-q_{12} q_{34}-2 q_{14} q_{34}-q_{23} q_{34}+q_{34}^{2}, q_{13}^{2}-q_{23}^{2}-q_{14} q_{24}-2 q_{13} q_{34}+q_{14} q_{34}+2 q_{23} q_{34}, \\
& q_{12} q_{14}-2 q_{14} q_{24}-q_{23} q_{24}-2 q_{24}^{2}+q_{12} q_{34}+2 q_{14} q_{34}+2 q_{23} q_{34}+2 q_{24} q_{34}-2 q_{34}^{2}, \\
& \left.q_{12} q_{13}+2 q_{13} q_{14}-q_{23}^{2}-q_{12} q_{24}-q_{13} q_{24}-2 q_{14} q_{24}-q_{23} q_{24}+3 q_{23} q_{34}+2 q_{24} q_{34}-2 q_{34}^{2}\right\rangle .
\end{aligned}
$$

From this, we easily find the octic equation of the trisecant surface $\mathcal{D}(X)$ :

$$
x_{1}^{7} x_{3}-2 x_{1}^{4} x_{2}^{3} x_{3}+x_{1} x_{2}^{6} x_{3}+2 x_{1}^{5} x_{2} x_{3}^{2}+2 x_{1}^{4} x_{2}^{2} x_{3}^{2}+2 x_{1}^{3} x_{2}^{3} x_{3}^{2}-2 x_{1}^{2} x_{2}^{4} x_{3}^{2}+\cdots
$$

This polynomial uses 136 of the $165=\left(\begin{array}{c}8+3 \\ 3\end{array}\right)$ monomials of degree eight.

The past few pages were devoted to specialized techniques that exploit the structure of a given curve $X$. Such techniques can be designed for all entries in Table 1. However, equally important are general purpose methods that work for all curves. We close this section by discussing the latter. The curve $X$ is given by its ideal $I=\left\langle f_{1}, f_{2}, \ldots, f_{k}\right\rangle$ in $\mathbb{R}\left[x_{1}, x_{2}, x_{3}, x_{4}\right]$.

The edge surface $\mathcal{E}(X)$ was already discussed in [33, 37]. We therefore focus on the other two ruled surfaces in Theorem 3.1. The easier among them is the tangential surface $\mathcal{T}(X)$. At any given point $p$ on the curve $X$, the tangent line is defined by the linear equations

$$
\left(\begin{array}{cccc}
\frac{\partial f_{1}}{\partial x_{1}}(p) & \frac{\partial f_{1}}{\partial x_{2}}(p) & \frac{\partial f_{1}}{\partial x_{3}}(p) & \frac{\partial f_{1}}{\partial x_{4}}(p) \\
\vdots & \vdots & \vdots & \vdots \\
\frac{\partial f_{k}}{\partial x_{1}}(p) & \frac{\partial f_{k}}{\partial x_{2}}(p) & \frac{\partial f_{k}}{\partial x_{3}}(p) & \frac{\partial f_{k}}{\partial x_{4}}(p)
\end{array}\right) \cdot\left(\begin{array}{c}
x_{1} \\
x_{2} \\
x_{3} \\
x_{4}
\end{array}\right)=\left(\begin{array}{l}
0 \\
0 \\
0 \\
0
\end{array}\right) .
$$


To find the polynomial $F$ defining $\mathcal{T}(X)$, we take a vector of variables $p=\left(y_{1}, y_{2}, y_{3}, y_{4}\right)$, and we augment $I$ with the constraints (14). This gives an ideal in $\mathbb{R}\left[x_{1}, x_{2}, x_{3}, x_{4}, y_{1}, y_{2}, y_{3}, y_{4}\right]$. From that ideal, we saturate and eliminate the variables $y_{1}, y_{2}, y_{3}, y_{4}$. The output is $\langle F\rangle$.

The trisecant surface $\mathcal{D}(X)$ will be represented by its curve $\mathcal{D}^{\ell}(X) \subset \operatorname{Gr}\left(1, \mathbb{P}^{3}\right)$. To compute this, we parameterize the line in $\mathbb{P}^{3}$ as in (2). Suppose for now that our curve is a complete intersection: $X=\left\{f_{1}=f_{2}=0\right\}$. We want the univariate polynomials $f_{1}(z(t))$ and $f_{2}(z(t))$ to have three common roots, i.e., their greatest common divisor (GCD) has degree $\geq 3$. This can be expressed using subresultants [1]. The vanishing of all subresultants of order $i=0, \ldots, r-1$ for two polynomials in $t$ means that their GCD has degree at least $r$. In our case, we form the ideal given by the subresultant coefficients of $f_{1}(z(t))$ and $f_{2}(z(t))$ of order 0,1 and 2 (together with the Plücker relation). The ideal of the trisecant curve $\mathcal{D}^{\ell}(X)$ is obtained by saturating by the ideal of the leading coefficients of $f_{1}(z(t))$ and $f_{2}(z(t))$.

This approach generalizes to the case when $X$ is not a complete intersection. Indeed, if $X$ is defined by $f_{1}, \ldots, f_{k}$, then we can use the same strategy to impose that $s_{1} f_{1}(z(t))+$ $\cdots+s_{k-1} f_{k-1}(z(t))$ and $f_{k}(z(t))$ have three roots in common for any choice of $s_{1}, \ldots, s_{k-1}$.

We conclude this section with an example that illustrates the last row of Table 1.

Example 3.12. Let $X$ be the smooth curve of degree 6 and genus 4 in $\mathbb{P}^{3}$ defined by

$$
x_{1}^{2}+x_{2}^{2}+x_{3}^{2}+x_{4}^{2}=x_{1}^{3}+x_{2}^{3}+x_{3}^{3}+x_{4}^{3}=0 .
$$

The above method easily yields the equation of degree 18 for the tangential surface $\mathcal{T}(X)$ :

$4 x_{1}^{12} x_{2}^{6}-12 x_{1}^{12} x_{2}^{5} x_{3}-12 x_{1}^{12} x_{2}^{5} x_{4}+21 x_{1}^{12} x_{2}^{4} x_{3}^{2}+\cdots+\underline{13770} x_{1}^{6} x_{2}^{4} x_{3}^{4} x_{4}^{4}+\cdots+24 x_{3}^{7} x_{4}^{11}+4 x_{3}^{6} x_{4}^{12}$.

This polynomial has 1094 terms. Its largest coefficient is underlined. A compact encoding is given by the 11 quadratic generators of the ideal of $\mathcal{T}^{\ell}(X)$. It is also easy to compute the quartic surface $\mathcal{D}(X)$, and it takes a little longer to compute the degree 54 curve $\mathcal{E}^{\ell}(X)$. $\diamond$

\section{Views of Surfaces}

We now turn to the visual events for a general surface $X$ in projective 3 -space $\mathbb{P}^{3}$. The six visual events associated with $X$ were mentioned in the Introduction in items (L) and $(\mathrm{M})$. We shall explain these events and how they give rise to the following five irreducible surfaces:

1. The flecnodal surface $\mathcal{F}(X)$ is the union of all lines $L$ with contact of order 4 at a point of $X$. In other words, the equation of $X$ restricted to $L$ has a root of multiplicity 4 .

2. The cusp crossing surface $\mathcal{C}(X)$ is the union of all lines $L$ with contact of order $3+2$ at two points of $X$, i.e., the equation for $X \cap L$ on $L$ has a triple root and a double root.

3. The tritangent surface $\mathcal{T}(X)$ is the union of all lines $L$ with contact of order $2+2+2$ at three points of $X$, i.e., the equation for $X \cap L$ on $L$ has three double roots.

4. The edge surface $\mathcal{E}(X)$ is the envelope of the bitangent planes of $X$. It is the union of all bitangent lines arising from these planes. This surface was denoted $\left(X^{[2]}\right)^{\vee}$ in [33]. 
5. The parabolic surface $\mathcal{P}(X)$ is the envelope of all tangent planes that have contact of order 3 with $X$. It is the union of all principal tangents at parabolic points [26, §A.1.2].

The following theorem characterizes the structure of the visual event surface for $X$ in $\mathbb{P}^{3}$.

Theorem 4.1. For a general surface $X$ of degree $d$ in $\mathbb{P}^{3}$, the visual event surface $\mathcal{V}(X)$ decomposes into the five components listed above. The degrees of these surfaces are:

$$
\begin{aligned}
& \operatorname{deg}(\mathcal{F}(X))=\quad 2 d(d-3)(3 d-2), \quad \text { [36, } \S 597] \text { and [25, Prop. 4.5] } \\
& \operatorname{deg}(\mathcal{C}(X))=d(d-3)(d-4)\left(d^{2}+6 d-4\right), \quad \text { [36, §598] and [25, Prop. 4.12] } \\
& \operatorname{deg}(\mathcal{T}(X))=\frac{1}{3} d(d-3)(d-4)(d-5)\left(d^{2}+3 d-2\right), \quad \text { [36, §599] and [25, Prop. 4.10] } \\
& \operatorname{deg}(\mathcal{E}(X))=d(d-2)(d-3)\left(d^{2}+2 d-4\right), \quad \text { [36, §613] and [25, Prop. 4.16] } \\
& \operatorname{deg}(\mathcal{P}(X))=\quad 2 d(d-2)(3 d-4) . \quad \text { [36, } \S 608] \text { and [25, Prop. 4.3] }
\end{aligned}
$$

We first learned these degree formulas from Petitjean's article [25]. We then discovered that all five formulas already appeared in Salmon's 1882 book [36]. The precise pointers to both sources are given above. In Section 5 we present new proofs that are self-contained, except for pointers to the textbook [12]. We here derive the first assertion in Theorem 4.1. that is, we justify the five irreducible surfaces. See the end of this section.

The five ruled surfaces in Theorem 4.1 are encoded by the curves shown in the last row in Figure 2. The surfaces $\mathcal{E}(X)$ and $\mathcal{P}(X)$ are developable, and are the duals of the singular loci shown on the left in Figure 2. The remaining three surfaces $\mathcal{T}(X), \mathcal{C}(X)$ and $\mathcal{F}(X)$ arise from the curves in the Grassmannian $\operatorname{Gr}\left(1, \mathbb{P}^{3}\right)$ seen on the right of that diagram.

The curves of lines and planes from Figure 2 capture both the local and multi-local features of the surface $X$. This is an advantage compared to the traditional approach for studying the appearance of surfaces based on differential geometry and singularity theory. In the computer vision literature [6, 26, 31, 32, 34], prominent local features of a surface were defined in terms of the euclidean Gauss map and the asymptotic spherical image. These are maps from the surface to the unit sphere $\mathbb{S}^{2}$, taking a point on $X_{\mathbb{R}}$ to its normal direction, or to the direction of one of its principal tangents. In our algebro-geometric setting, the role of $\mathbb{S}^{2}$ is played by the dual surface $X^{\vee} \subset\left(\mathbb{P}^{3}\right)^{*}$ and the principal tangent surface $P T(X) \subset \operatorname{Gr}\left(1, \mathbb{P}^{3}\right)$. These surfaces carry much more information than the unit sphere $\mathbb{S}^{2}$.

Consider now the projection $\pi_{z}: X \subset \mathbb{P}^{3} \rightarrow \mathbb{P}^{2}$ from a center $z \in \mathbb{P}^{3} \backslash X$. The following result, analogous to Proposition 3.2. describes intrinsic realizations of the contour $C_{z}(X)$.

Proposition 4.2. The contour $C_{z}(X)$ of our surface $X$ is projectively equivalent to the curve $\alpha(z) \cap \operatorname{Hur}(X)$ in the Grassmannian $\operatorname{Gr}\left(1, \mathbb{P}^{3}\right)$. The curve $\left(C_{z}(X)\right)^{\vee}$ in $\left(\mathbb{P}^{2}\right)^{*}$ that is dual to the contour is projectively equivalent to the curve $z^{\vee} \cap X^{\vee}$ in the dual projective space $\left(\mathbb{P}^{3}\right)^{*}$.

We now describe the singularities of the curve $C_{z}(X)$ that arises by projecting $X$ from $z$. Given a point $u \in C_{z}(X)$, we write $L_{u}=\pi_{z}^{-1}(u) \in \operatorname{Gr}\left(1, \mathbb{P}^{3}\right)$ for its fiber under $\pi_{z}$. For a general viewpoint $z$, the plane $\alpha(z)$ intersects the Hurwitz threefold $\operatorname{Hur}(X)$ transversely:

- If $L_{u}$ is a general point of $\operatorname{Hur}(X)$, then $u$ is a smooth point of the curve $C_{z}(X)$.

- If $L_{u}$ is a general point of $\operatorname{Bit}(X) \subset \operatorname{Hur}(X)$, then $u$ is a simple node of $C_{z}(X)$. 
- If $L_{u}$ is a general point of $\mathrm{PT}(X) \subset \operatorname{Hur}(X)$, then $u$ is a simple cusp of $C_{z}(X)$.

These singularities exist for any viewpoint $z$, since $\alpha(z)$ always intersects the surfaces $\operatorname{Bit}(X)$ and $\operatorname{PT}(X)$ in $\operatorname{Gr}\left(1, \mathbb{P}^{3}\right)$. It is also interesting to learn (e.g. from [31]) that if $L_{u}$ is a (non-principal) tangent line at a parabolic point, then $u$ is a flex point of $C_{z}(X)$. We are interested in higher order singularities seen in the image curve for special viewpoints:

$(\mathcal{T})$ If $L_{u}$ is a tritangent line, then $u$ is a triple point. This is a triple point event.

$(\mathcal{C})$ If $L_{u}$ is a principal bitangent, then $C_{z}(X)$ has a smooth branch and a cuspidal branch that meet at $u$. This is a cusp crossing event.

$(\mathcal{F})$ If $L_{u}$ is a flecnodal line, then $u$ is the limit of two cusps and a node, i.e., an infinitesimal change of the viewpoint produces two cusps and a node. This is a swallowtail event.

$(\mathcal{E})$ If $L_{u}$ is a bitangent line on a bitangent plane, then $u$ is a tacnode. It is obtained as the limit of two smooth branches coming together at $u$. This is a tangent crossing event.

$(\mathcal{P})$ If $L_{u}$ is the principal tangent at a parabolic point $p$, then, over the real numbers, two behaviors are possible: either $u$ is an isolated node, which corresponds to a lip event, or $u$ is a tacnode, obtained as the limit of two cusps, which is a beak-to-beak event.

The triple point, cusp crossing, and tangent crossing events are multi-local. The six visual events are shown in Figure 4. Detailed renderings of these pictures are ubiquitous in the relevant computer vision literature. For instance, see [26, Figures 5 and 6], and Figures 13.20 through 13.25 in the textbook [14]. For a fixed general surface $X$ in $\mathbb{P}^{3}$, the locus of exceptional viewpoints consists of the five ruled surfaces $\mathcal{F}(X), \mathcal{C}(X), \mathcal{T}(X), \mathcal{E}(X)$ and $\mathcal{P}(X)$. These were defined both in Figure 2 and at the beginning of Section 4.

We now briefly explain how to distinguish the two possible local behaviors (lip versus beak-to-beak) of the contour when the viewpoint $z$ belongs to the parabolic surface $\mathcal{P}(X)$. As argued in Section 2, the parabolic surface $\mathcal{P}(X)$ is a developable surface, since it is dual to the curve $\mathcal{P}^{p}(X)$ in $\left(\mathbb{P}^{3}\right)^{*}$. In particular, all principal tangents at parabolic points are the tangents of the edge of regression curve, denoted by $E(\mathcal{P}(X))$. This allows us to associate each parabolic point $x$ with another point $e_{x}$, where the principal tangent at $x$ is tangent to $E\left(\mathcal{P}(X)\right.$ ). In real projective 3 -space, the complement of $\left\{x, e_{x}\right\}$ in that line has two connected components. The distinction between lip and beak-to-beak is made by which of these two components the viewpoint $z$ belongs to. This was shown in [30, Theorem 4.10].

We next offer an informal discussion that should provide an intuitive understanding of our five event surfaces. The following are some real life situations where these events can actually be observed. We encourage our readers to look at the world from multiple viewpoints, and to then spot the six pictures of Figure 4. Do look carefully at the objects that surround you.

We first note that cuspidal and nodal singularities of image contours are stable features, which are visible in most surfaces. Nodes occur whenever occlusions create discontinuities in the contour. Cusps only appear for non-convex objects. For instance, they can be observed on the folds of a piece of cloth. From an exceptional viewpoint, it is possible that several 

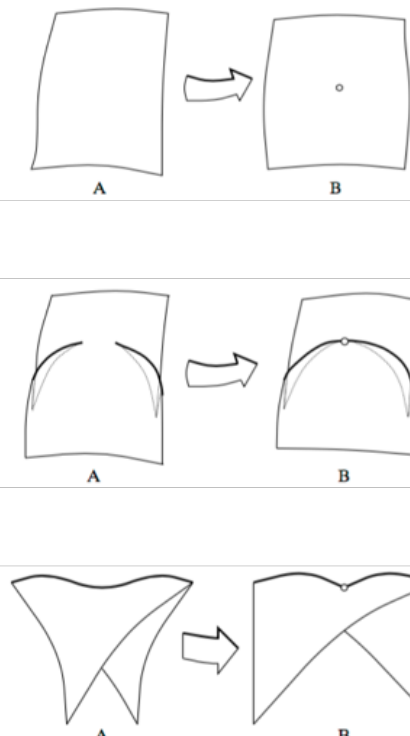

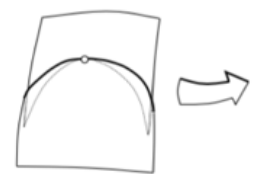

B
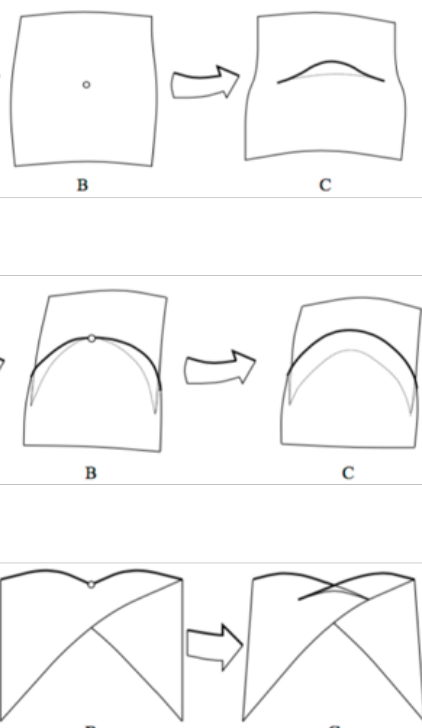
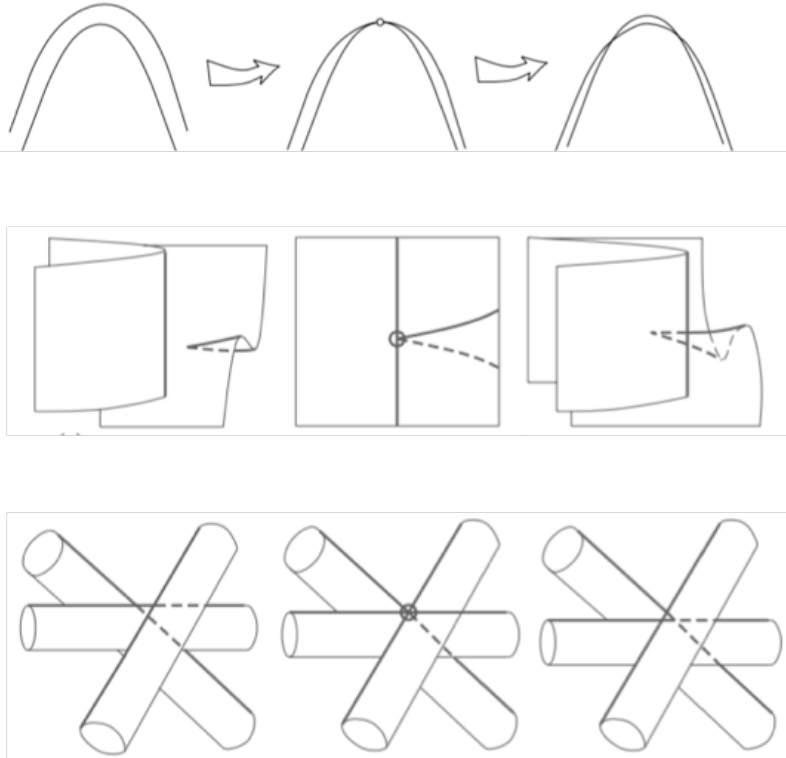

Figure 4: The catalogue of visual events for the projections of a smooth surface from a viewpoint that moves. The local events (left, from top to bottom) are lip, beak-to-beak, swallowtail. The multi-local events (right, from top to bottom) are tangent crossing, cusp crossing, triple point. Reprinted from [24] with permission of Springer.

of these singularities occur along the same visual ray. This gives rise to a multi-local visual event (cusp crossing, tangent crossing, or triple points). Try it with a napkin or towel.

The three local events on the left in Figure 4 are more complicated. Videos of these events and their corresponding ruled surfaces are available at

wWw.mis.mpg.de/nlalg/videos/changingviews

It takes some practice to discover the local events in the real world. Here are some concrete examples that we found helpful:

- Lip event: If we observe a small hill from a high aerial viewpoint (say, from a hot air balloon), then all points on the ground are visible. The hill does not generate an image contour. However, as we descend closer to the ground, the profile of the hill suddenly becomes visible in the contour. This qualitative change of appearance is a lip event.

- Beak-to-beak. Observe a glass bottle from the bottom, with your eye close to the base. You see a part of the contour generated by the convex region where the sectional diameter of the bottle decreases. Now, tilt the bottle slowly towards its upright position. At some point, you see a complete path from the base to the top of the bottle. Previously your view had been blocked. This is a beak-to-beak event. Contrary to the lip event, the contour does not disappear at the transition point, but it breaks into two pieces.

- Swallowtail. The traditional drawing of a (transparent) torus presents two swallowtails. We see both cuspidal and nodal singularities in the contour [34, Fig. 2]. As we rotate the torus, a visual event occurs, and these singularities disappear. Try it with a bagel. 
After having presented an intuitive descriptions of visual events, we now sketch a formal argument which shows that the list of visual events in Theorem 4.1 is indeed exhaustive.

Proof sketch for the first part of Theorem 4.1. Let $u \in C_{z}(X)$ and $L_{u}:=\pi_{z}^{-1}(u)$. Platonova [30, Main Theorem] characterizes all possible local singularities in the contour curve. In particular, she shows in the case $L_{u} \in \mathrm{PT}(X)$ that $u$ is not a simple cusp on its branch of the contour curve if and only if $L_{u}$ is either a flecnodal line or the unique principal tangent at a parabolic point. Hence, we only have to characterize the possible multi-local events. We argued above that tritangent lines and principal bitangent lines do not yield simple nodes in the contour. The final observation is that a line $L_{u}$ with contact order exactly two at exactly two distinct points of the surface $X$ projects to a simple node $u$ if and only if $L_{u}$ is not contained in a bitangent plane to $X$ which is tangent at the same two points as $L_{u}$.

\section{$5 \quad$ Intersection Theory}

In this section we derive the degrees of $\mathcal{E}^{p}(X), \mathcal{P}^{p}(X), \mathcal{P}(X)$ and $\mathcal{F}(X)$, and we sketch the relevant ideas for $\mathcal{E}(X), \mathcal{C}(X)$ and $\mathcal{T}(X)$. We found Petitjean's proofs in [25] to be lengthy and hard. They require a full understanding of Colley's multiple point theory [8, 9], and several of the steps are left out. By contrast, the derivations in Salmon's book [36] are inspiring but they lack the rigor of 20th century intersection theory.

The exposition that follows refers to the textbook by Eisenbud and Harris [12]. We believe that students of that book will find this section to be useful as supplementary reading.

We have discussed that the surfaces $\mathcal{E}(X)$ and $\mathcal{P}(X)$ are represented by their dual curves in $\left(\mathbb{P}^{3}\right)^{*}$. These are the irreducible components in the singular locus of the dual surface $X^{\vee}$.

Proposition 5.1. The degrees of the curves dual to the edge and parabolic surface of a general surface $X$ of degree $d \geq 4$ or $d \geq 3$, respectively, are

$\operatorname{deg}\left(\mathcal{E}^{p}(X)\right)=\frac{1}{2} d(d-1)(d-2)\left(d^{3}-d^{2}+d-12\right) \quad$ and $\quad \operatorname{deg}\left(\mathcal{P}^{p}(X)\right)=4 d(d-1)(d-2)$.

Proof. We count the bitangent and parabolic planes that contain a general point $z \in \mathbb{P}^{3}$. Consider the branch curve $C_{z}(X) \subset \mathbb{P}^{2}$. A bitangent plane to $X$ containing $z$ maps onto a bitangent line of $C_{z}(X)$. A tangent plane to $X$ at a parabolic point that contains $z$ maps onto a flex line of $C_{z}(X)$. These correspond, respectively, to the nodes and the cusps of the dual curve $C_{z}(X)^{\vee}$ in $\left(\mathbb{P}^{2}\right)^{*}$. We use Plücker's formula [10, Thm. 1.2.7]: for an irreducible plane curve of degree $D$ with $\nu$ nodes, $\kappa$ cusps and no other singularities, its dual has degree $D(D-1)-2 \nu-3 \kappa$. The genus is $\frac{1}{2}(D-1)(D-2)-\nu-\kappa$, by the degree-genus formula.

The ramification curve of the projection $\pi_{z}$ has degree $d(d-1)$ since it is defined by the equation $f$ of $X$ and its derivative $g(x):=\sum_{i=1}^{4} z_{i} \frac{\partial f}{\partial x_{i}}$. Hence $C_{z}(X)$ has degree $d(d-1)$ as well. The degree of the dual curve $\left(C_{z}(X)\right)^{\vee} \subset\left(\mathbb{P}^{2}\right)^{*}$ equals the degree of the dual surface $X^{\vee}$ (see [22, proof of Thm. 4.3] for details). This common degree is $d(d-1)^{2}$.

We denote by $\nu_{1}$ the number of nodes in $C_{z}(X)$, which is the number of bitangent lines to $X$ passing through the general point $z$. The number $\kappa_{1}$ of cusps in $C_{z}(X)$ is the number 
of principal tangents to $X$ passing through $z$. The set of points $x \in X$ that have such a principal tangent through $z$ is the zero locus of the polynomials $f, g$ and $h(x):=\sum_{i=1}^{4} z_{i} \frac{\partial g}{\partial x_{i}}=$ $z H_{f}(x) z^{T}$. Hence, $\kappa_{1}=d(d-1)(d-2)$. Plücker's formula for $C_{z}(X)$ tells us that

$$
\begin{aligned}
d(d-1)^{2}=\operatorname{deg}\left(\left(C_{z}(X)\right)^{\vee}\right) & =\operatorname{deg}\left(C_{z}(X)\right)\left(\operatorname{deg}\left(C_{z}(X)\right)-1\right)-2 \nu_{1}-3 \kappa_{1} \\
& =d(d-1)(d(d-1)-1)-2 \nu_{1}-3 \kappa_{1} .
\end{aligned}
$$

Thus the number of nodes of the branch curve $C_{z}(X)$ equals $\nu_{1}=\frac{1}{2} d(d-1)(d-2)(d-3)$. We can also apply Plücker's formula to the dual plane curve $C_{z}(X)^{\vee}$ to derive

$$
\begin{aligned}
d(d-1)=\operatorname{deg}\left(C_{z}(X)\right) & =\operatorname{deg}\left(\left(C_{z}(X)\right)^{\vee}\right)\left(\operatorname{deg}\left(\left(C_{z}(X)\right)^{\vee}\right)-1\right)-2 \nu_{2}-3 \kappa_{2} \\
& =d(d-1)^{2}\left(d(d-1)^{2}-1\right)-2 \nu_{2}-3 \kappa_{2},
\end{aligned}
$$

where $\nu_{2}=\operatorname{deg}\left(\mathcal{E}^{p}(X)\right)$ and $\kappa_{2}=\operatorname{deg}\left(\mathcal{P}^{p}(X)\right)$, as argued above. The dual curves $C_{z}(X)$ and $\left(C_{z}(X)\right)^{\vee}$ have the same geometric genus. By the degree-genus formula, it is

$$
\frac{1}{2}(d(d-1)-1)(d(d-1)-2)-\nu_{1}-\kappa_{1}=\frac{1}{2}\left(d(d-1)^{2}-1\right)\left(d(d-1)^{2}-2\right)-\nu_{2}-\kappa_{2} .
$$

Solving the equations (15) and (16) for $\nu_{2}$ and $\kappa_{2}$ leads to the formulas in Proposition 5.1.

This proof was entirely classical. By contrast, we derive the degrees of the visual event surfaces using modern intersection theory [12. At the heart of intersection theory lies the Chow ring. For any irreducible projective variety $Y$ and any $c \in\{0, \ldots, \operatorname{dim} Y\}$, we denote by $\mathrm{CH}^{c}(Y)$ the free abelian group generated by the set of irreducible closed subvarieties of $Y$ with codimension $c$, modulo rational equivalence. We call elements in $\mathrm{CH}^{c}(Y)$ codimension- $c$ cycles. The rational equivalence class of a subvariety $Z \subset Y$ is denoted by $[Z]$. We denote the class of a point in $Y$ by $* \in \mathrm{CH}^{\operatorname{dim} Y}(Y)$, whenever this is well-defined.

Theorem/Definition 5.2. [12, Thm-Def 1.5] If $Y$ is a smooth projective variety, then there is a unique product structure on $\mathrm{CH}(Y)=\bigoplus_{c=0}^{\operatorname{dim} Y} \mathrm{CH}^{c}(Y)$ which satisfies the condition:

If two subvarieties $Z_{1}, Z_{2} \subset Y$ are generically transverse, then $\left[Z_{1}\right]\left[Z_{2}\right]=\left[Z_{1} \cap Z_{2}\right]$.

By generically transverse we mean that every irreducible component of $Z_{1} \cap Z_{2}$ contains a point where $Z_{1}$ and $Z_{2}$ are transverse. This makes $\mathrm{CH}(Y)$ into an associative commutative graded ring, called the Chow ring of $Y$.

Example 5.3. [12, Theorem 2.1] The Chow ring of projective 3 -space is $\mathrm{CH}\left(\mathbb{P}^{3}\right)=\mathbb{Z}[h] /\left\langle h^{4}\right\rangle$, where $h \in \mathrm{CH}^{1}\left(\mathbb{P}^{3}\right)$ is the (rational equivalence) class of a plane in $\mathbb{P}^{3}$.

Example 5.4. [12, Theorem 3.10] The Chow ring of the Grassmannian $\operatorname{Gr}\left(1, \mathbb{P}^{3}\right)$ is a graded $\mathbb{Z}$-algebra with Hilbert function $(1,1,2,1,1)$. The graded components are the free $\mathbb{Z}$-modules

$$
\begin{aligned}
& \mathrm{CH}^{0}\left(\operatorname{Gr}\left(1, \mathbb{P}^{3}\right)\right)=\mathbb{Z} \cdot\left[\operatorname{Gr}\left(1, \mathbb{P}^{3}\right)\right], \quad \mathrm{CH}^{1}\left(\operatorname{Gr}\left(1, \mathbb{P}^{3}\right)\right)=\mathbb{Z} \gamma_{1}, \quad \mathrm{CH}^{2}\left(\operatorname{Gr}\left(1, \mathbb{P}^{3}\right)\right)=\mathbb{Z} \gamma_{1}^{2} \oplus \mathbb{Z} \gamma_{2}, \\
& \mathrm{CH}^{3}\left(\operatorname{Gr}\left(1, \mathbb{P}^{3}\right)\right)=\mathbb{Z} \gamma_{1} \gamma_{2}, \mathrm{CH}^{4}\left(\operatorname{Gr}\left(1, \mathbb{P}^{3}\right)\right) \quad=\mathbb{Z} *,
\end{aligned}
$$


where $\gamma_{1}$ represents the threefold of all lines that meet a given line, and $\gamma_{2}$ represents the surface of all lines that lie in a given plane. The multiplicative structure is given by

$$
\gamma_{1}^{3}=2 \gamma_{1} \gamma_{2}, \quad \gamma_{2}^{2}=*, \quad \gamma_{1}^{2} \gamma_{2}=*, \quad \gamma_{1}^{4}=2 *
$$

Further, the class $\gamma_{1}^{2}-\gamma_{2}$ represents the surface of all lines passing through a given point. $\diamond$

Example 5.5. [12, Proposition 9.10] The universal line $\Phi=\left\{(x, L) \in \mathbb{P}^{3} \times \operatorname{Gr}\left(1, \mathbb{P}^{3}\right) \mid\right.$ $x \in L\}$ is a 5 -dimensional smooth projective variety. The Chow ring of this variety equals $\mathrm{CH}(\Phi)=\mathrm{CH}\left(\operatorname{Gr}\left(1, \mathbb{P}^{3}\right)\right)[H] /\left\langle H^{2}-\gamma_{1} H+\gamma_{2}\right\rangle$. Here, the class $H \in \mathrm{CH}^{1}(\Phi)$ represents the preimage of a plane in $\mathbb{P}^{3}$ under the projection $\Phi \rightarrow \mathbb{P}^{3}$ onto the first factor.

Proposition 5.6. For a general surface $X$ of degree $d \geq 4$, the degrees of the flecnodal surface $\mathcal{F}(X)$ and the flecnodal curve $F$ on $X$ are

$$
\operatorname{deg}(\mathcal{F}(X))=2 d(d-3)(3 d-2) \quad \text { and } \quad \operatorname{deg}(F)=d(11 d-24) .
$$

Proof. Let $X_{\Phi}^{4} \subset \Phi$ be the incidence variety of pairs $(x, L)$ such that the line $L$ has contact of order at least 4 at the point $x \in X$. The degree of the flecnodal surface $\mathcal{F}(X)$ is the number of intersections of $\mathcal{F}(X)$ with a general line. This is the number of pairs $(x, L) \in X_{\Phi}^{4}$ such that $L$ meets a general line. In particular, we have $\left[X_{\Phi}^{4}\right] \cdot \gamma_{1}=\operatorname{deg}(\mathcal{F}(X)) *$ in $\operatorname{CH}(\Phi)$.

We shall compute $\left[X_{\Phi}^{4}\right] \in \mathrm{CH}^{4}(\Phi)$ via Chern classes. The top Chern class $c_{r}(\mathcal{E}) \in \mathrm{CH}^{r}(Y)$ of a vector bundle $\mathcal{E}$ of rank $r$ on a smooth variety $Y$ with a global section $\sigma: Y \rightarrow \mathcal{E}$ is the class of the vanishing locus of $\sigma$. This definition is independent of the chosen global section. Fix any vector bundle $\mathcal{E}$ on $\Phi$ and any integer $m \in \mathbb{N}$. By [12, Theorem 11.2], there is a new vector bundle $\mathcal{J}_{\Phi / \operatorname{Gr}\left(1, \mathbb{P}^{3}\right)}^{m}(\mathcal{E})$ on $\Phi$ whose fiber at $(x, L)$ is the space of all germs of sections of $\left.\mathcal{E}\right|_{\{(y, L) \in \Phi\}}$ modulo those that vanish to order $\geq m+1$ at $(x, L)$. This is called the bundle of relative principal parts or the relative jet bundle. We shall compute its top Chern class.

Let now $\mathcal{E}$ be the pullback to $\Phi$ of the line bundle $\mathcal{O}_{\mathbb{P}^{3}}(d)$. A global section of $\mathcal{E}$ is given by the homogeneous polynomial in $x_{1}, x_{2}, x_{3}, x_{4}$ of degree $d$ that defines $X$ in $\mathbb{P}^{3}$. Restricting this polynomial to the line $L$ gives a global section of $\left.\mathcal{E}\right|_{\{(y, L) \in \Phi\}}$. That global section vanishes at $(x, L)$ if and only if $L$ has contact of order at least $m+1$ at $x \in X$. Hence, the top Chern class of $\mathcal{J}_{\Phi / \operatorname{Gr}\left(1, \mathbb{P}^{3}\right)}^{m}(\mathcal{E})$ is the class of the subvariety of all pairs $(x, L)$ in $\Phi$ such that $L$ has contact of order at least $m+1$ at $x \in X$. In particular, $c_{4}\left(\mathcal{J}_{\Phi / \operatorname{Gr}\left(1, \mathbb{P}^{3}\right)}^{3}(\mathcal{E})\right)=\left[X_{\Phi}^{4}\right]$.

In addition, we see from Theorem 11.2 in [12] that $\mathcal{J}_{\Phi / \operatorname{Gr}\left(1, \mathbb{P}^{3}\right)}^{m}(\mathcal{E})$ agrees locally with

$$
\mathcal{E} \oplus\left(\mathcal{E} \otimes \Omega_{\Phi / \operatorname{Gr}\left(1, \mathbb{P}^{3}\right)}\right) \oplus\left(\mathcal{E} \otimes \operatorname{Sym}^{2} \Omega_{\Phi / \operatorname{Gr}\left(1, \mathbb{P}^{3}\right)}\right) \oplus \cdots \oplus\left(\mathcal{E} \otimes \operatorname{Sym}^{m} \Omega_{\Phi / \operatorname{Gr}\left(1, \mathbb{P}^{3}\right)}\right)
$$

where $\Omega_{\Phi / \operatorname{Gr}\left(1, \mathbb{P}^{3}\right)}$ is the relative cotangent bundle, which has rank 1 in our case. We compute the top Chern class of $\mathcal{J}_{\Phi / \operatorname{Gr}\left(1, \mathbb{P}^{3}\right)}^{m}(\mathcal{E})$ from its representation $(17)$. From [12, p. 395] we have $c_{1}\left(\operatorname{Sym}^{m} \Omega_{\Phi / \operatorname{Gr}\left(1, \mathbb{P}^{3}\right)}\right)=m\left(\gamma_{1}-2 H\right)$ in $\mathrm{CH}^{1}(\Phi)$. Since the equation $f$ of $X$ gives a global section of $\mathcal{E}$, we further have $c_{1}(\mathcal{E})=d H$ in $\mathrm{CH}^{1}(\Phi)$. The top Chern class of the tensor product of two line bundles is the sum of their top Chern classes [12, Prop. 5.17]. Hence

$$
c_{1}\left(\mathcal{E} \otimes \operatorname{Sym}^{i} \Omega_{\Phi / \operatorname{Gr}\left(1, \mathbb{P}^{3}\right)}\right)=(d-2 i) H+i \gamma_{1} \quad \text { in } \quad \mathrm{CH}^{1}(\Phi) \quad \text { for } \quad i=0,1,2, \ldots, m .
$$


Finally, Whitney's sum formula [12, Theorem 5.3] says that the top Chern class of a direct sum of vector bundles is the product of the top Chern classes of the summands. This implies

$$
c_{m+1}\left(\mathcal{J}_{\Phi / \operatorname{Gr}\left(1, \mathbb{P}^{3}\right)}^{m}(\mathcal{E})\right)=\prod_{i=0}^{m}\left((d-2 i) H+i \gamma_{1}\right) \quad \text { in } \quad \mathrm{CH}^{m+1}(\Phi) .
$$

We have $H^{4}=0$, by Example 5.3 and $H^{3} \gamma_{1}^{2}=*$, since exactly one line meets two general lines and passes through a given point. Finally, $H^{2} \gamma_{1}^{3}=2 *$ and $H \gamma_{1}^{4}=2 *$, since exactly two lines meet four general lines in $\mathbb{P}^{3}$. Putting these pieces together, we get the desired formula

$$
\begin{aligned}
\operatorname{deg}(\mathcal{F}(X)) * & =\left[X_{\Phi}^{4}\right] \cdot \gamma_{1}=c_{4}\left(\mathcal{J}_{\Phi / \operatorname{Gr}\left(1, \mathbb{P}^{3}\right)}(\mathcal{E})\right) \cdot \gamma_{1}=\gamma_{1} \prod_{i=0}^{3}\left((d-2 i) H+i \gamma_{1}\right) \\
& =\left(6 d^{3}-44 d^{2}+72 d\right) H^{3} \gamma_{1}^{2}+\left(11 d^{2}-36 d\right) H^{2} \gamma_{1}^{3}+6 d H \gamma_{1}^{4}=2 d(d-3)(3 d-2) * .
\end{aligned}
$$

An analogous computation yields the degree of the flecnodal curve $F$ on the surface $X$. We find that $\operatorname{deg}(F) *=\left[X_{\Phi}^{4}\right] \cdot H=H \prod_{i=0}^{3}\left((d-2 i) H+i \gamma_{1}\right)=d(11 d-24) *$.

Proposition 5.7. The degree of the parabolic surface of a general surface $X$ of degree $d \geq 3$ is

$$
\operatorname{deg}(\mathcal{P}(X))=2 d(d-2)(3 d-4) .
$$

Proof. We consider the incidence variety $P_{\Phi}^{3}$ that consists of all pairs $(x, L)$ in $\Phi$ with the property that $x$ is parabolic on $X$ and $L$ has contact order at least 3 at $x \in X$. Settheoretically, this is the intersection of the variety $X_{\Phi}^{3} \subset \Phi$ of pairs $(x, L)$ such that $L$ is a principal tangent to $X$ at $x$ with the variety $P_{\Phi}^{1} \subset \Phi$ of pairs $(x, L)$ such that $x$ is parabolic on $X$. Since $\operatorname{codim}_{\Phi} P_{\Phi}^{3}=4<3+2=\operatorname{codim}_{\Phi} X_{\Phi}^{3}+\operatorname{codim}_{\Phi} P_{\Phi}^{1}$, we cannot simply multiply their classes in $\mathrm{CH}(\Phi)$ to get the class of $P_{\Phi}^{3}$.

We shall compute all relevant classes in $\mathrm{CH}\left(\Phi_{X}\right)$, where $\Phi_{X}$ is the variety of pairs $(x, L) \in$ $\Phi$ with $x \in X$. The varieties $X_{\Phi}^{3}$ and $P_{\Phi}^{1}$ intersect with the expected codimension in $\Phi_{X}$, although this intersection is not transverse. It is difficult to describe the Chow ring of $\Phi_{X}$. We find some generators and relations of $\mathrm{CH}\left(\Phi_{X}\right)$ by taking pullbacks of elements in $\mathrm{CH}(\Phi)$ under the inclusion $\Phi_{X} \hookrightarrow \Phi$. Recall that the pullback of a class $[Z]$ under a nice enough morphism $f$ between varieties is $\left[f^{-1}(Z)\right]$, by [12, Thm. 1.23]. We denote by $E \in \mathrm{CH}^{1}\left(\Phi_{X}\right)$ the pullback of the hyperplane class $H \in \mathrm{CH}^{1}(\Phi)$ and by $\Gamma_{i} \in \mathrm{CH}\left(\Phi_{X}\right)$ the pullback of $\gamma_{i} \in \mathrm{CH}(\Phi)$ for $i \in\{1,2\}$.

By definition, $P_{\Phi}^{1}$ is the preimage of the parabolic curve $P \subset X$ under the map $\Phi_{X} \rightarrow X$. The parabolic curve $P$ is the intersection of $X$ with the Hessian surface of degree $4(d-2)$. Thus, the class of $P$ in $\mathrm{CH}(X)$ is $4(d-2) e$, where $e \in \mathrm{CH}^{1}(X)$ is the pullback of the hyperplane class $h \in \mathrm{CH}^{1}\left(\mathbb{P}^{3}\right)$ under the inclusion $X \hookrightarrow \mathbb{P}^{3}$. Since $E \in \mathrm{CH}^{1}\left(\Phi_{X}\right)$ is also the pullback of $e \in \mathrm{CH}^{1}(X)$ under the projection $\Phi_{X} \rightarrow X$, we have $\left[P_{\Phi}^{1}\right]=4(d-2) E \in \mathrm{CH}^{1}\left(\Phi_{X}\right)$. We compute $\left[X_{\Phi}^{3}\right]$ in the Chow ring of $\Phi$ using the formula (17) with $m=2$ :

$$
\left[X_{\Phi}^{3}\right]=c_{3}\left(\mathcal{J}_{\Phi / \operatorname{Gr}\left(1, \mathbb{P}^{3}\right)}^{2}(\mathcal{E})\right)=\prod_{i=0}^{2}\left((d-2 i) H+i \gamma_{1}\right)=d H\left(\left(d^{2}-6 d+8\right) H^{2}+(3 d-8) H \gamma_{1}+2 \gamma_{1}^{2}\right) .
$$

The right hand side lives in the Chow ring of the 5-dimensional variety $\Phi$ and we pull it back to the Chow ring of its 4-dimensional subvariety $\Phi_{X}$. That pullback is the codimension-3 
cycle $d E \beta$, where $\beta:=\left(d^{2}-6 d+8\right) E^{2}+(3 d-8) E \Gamma_{1}+2 \Gamma_{1}^{2} \in \mathrm{CH}^{2}\left(\Phi_{X}\right)$. Since pullback preserves codimension, $\left[X_{\Phi}^{3}\right] \in \mathrm{CH}^{2}\left(\Phi_{X}\right)$ cannot be equal to $d E \beta \in \mathrm{CH}^{3}\left(\Phi_{X}\right)$. Instead [12, Theorem 13.7] tells us that $d E \beta=\left[X_{\Phi}^{3}\right] \cdot c_{1}\left(\mathcal{N}_{\Phi_{X} / \Phi}\right) \in \mathrm{CH}^{3}\left(\Phi_{X}\right)$, where $\mathcal{N}_{\Phi_{X} / \Phi}$ is the normal bundle of $\Phi_{X}$ in $\Phi$. By [12, Prop.-Def. 6.15], we have $c_{1}\left(\mathcal{N}_{\Phi_{X} / \Phi}\right)=d E$ and $d E \beta=d E\left[X_{\Phi}^{3}\right]$.

We cannot yet say that $\left[X_{\Phi}^{3}\right]=\beta$ in $\mathrm{CH}^{2}\left(\Phi_{X}\right)$ because multiplication with $E$ has nontrivial kernel. However, since $E$ is a factor of $\left[P_{\Phi}^{1}\right]$, we conclude that $\left[X_{\Phi}^{3}\right]\left[P_{\Phi}^{1}\right]=4(d-2) E \beta$ in the Chow ring of $\Phi_{X}$. Since a general point on $X$ has two principal tangent lines, the intersection multiplicity of the varieties $P_{\Phi}^{1}$ and $X_{\Phi}^{3}$ is 2. Therefore, by [12, Theorem 1.26], we have $\left[P_{\Phi}^{3}\right]=\frac{1}{2}\left[X_{\Phi}^{3}\right]\left[P_{\Phi}^{1}\right]=2(d-2) E \beta$ in $\mathrm{CH}\left(\Phi_{X}\right)$. As in the case of flecnodal lines, the degree of the parabolic surface $\mathcal{P}(X)$ is the number of points in the 0-dimensional cycle

$$
\left[P_{\Phi}^{3}\right] \cdot \Gamma_{1}=2(d-2)\left(\left(d^{2}-6 d+8\right) E^{3} \Gamma_{1}+(3 d-8) E^{2} \Gamma_{1}^{2}+2 E \Gamma_{1}^{3}\right) .
$$

Finally, we use the pushforward of the inclusion $i: \Phi_{X} \hookrightarrow \Phi$ to express the above monomials in the point class $*$ of $\mathrm{CH}(\Phi)$. The pushforward $f_{*}$ of a proper morphism $f$ maps $[Z]$ to 0 if $\operatorname{dim}(f(Z))<\operatorname{dim}(Z)$, and otherwise to $\nu[f(Z)]$ where $\nu \in \mathbb{Z}$ denotes the degree of the restricted map $\left.f\right|_{Z}: Z \rightarrow f(Z)$. Using the push-pull formula [12, Theorem 1.23], we derive $i_{*}\left(E^{2} \Gamma_{1}^{2}\right)=i_{*}\left(i^{*}\left(H^{2} \gamma_{1}^{2}\right) \cdot\left[\Phi_{X}\right]\right)=H^{2} \gamma_{1}^{2} \cdot i_{*}\left(\left[\Phi_{X}\right]\right)=d H^{3} \gamma_{1}^{2}=d *$. Similarly, we get $i_{*}\left(E \Gamma_{1}^{3}\right)=2 d *$ and $i_{*}\left(E^{3} \Gamma_{1}\right)=0$. Hence, $\operatorname{deg}(\mathcal{P}(X)) *=i_{*}\left(\left[P_{\Phi}^{3}\right] \cdot \Gamma_{1}\right)=2 d(d-2)(3 d-4) *$.

Proposition 5.8. The degree of the edge surface of a general surface $X$ of degree $d \geq 4$ is

$$
\operatorname{deg}(\mathcal{E}(X))=d(d-2)(d-3)\left(d^{2}+2 d-4\right)
$$

Proof sketch. We describe an idea for computing the degree of $\mathcal{E}(X)$ with a mix of classical methods and intersection theory, similar to Petitjean's approach [25, Prop. 4.14-4.16]. The Gauss map $\gamma: X \rightarrow\left(\mathbb{P}^{3}\right)^{*}$ assigns to each point $x \in X$ the tangent plane to $X$ at $x$. The preimage of $\mathcal{E}^{p}(X)$ under $\gamma$ is the node-couple curve $C \subset X$, and the restriction $\left.\gamma\right|_{C}: C \rightarrow$ $\mathcal{E}^{p}(X)$ is a 2 -to- 1 covering of $\mathcal{E}^{p}(X)$. From this we get that $\gamma_{*}([C])=2\left[\mathcal{E}^{p}(X)\right]$ in $\mathrm{CH}\left(\left(\mathbb{P}^{3}\right)^{*}\right)$.

We can compute the class $[C]$ in $\mathrm{CH}^{1}(X)$ as follows. Let us denote by $h^{*} \in \mathrm{CH}^{1}\left(\left(\mathbb{P}^{3}\right)^{*}\right)$ the class of a hyperplane in $\left(\mathbb{P}^{3}\right)^{*}$, and by $e^{*} \in \mathrm{CH}^{1}(X)$ the pullback of $h^{*}$ under the Gauss map $\gamma$. Since $e \in \mathrm{CH}^{1}(X)$ (which we defined as the pullback of the hyperplane class $h \in \mathrm{CH}^{1}\left(\mathbb{P}^{3}\right.$ ) under the inclusion $j: X \hookrightarrow \mathbb{P}^{3}$ ) generates $\mathrm{CH}^{1}(X)$, we know that $e^{*}=\alpha e$ for some $\alpha \in \mathbb{Z}$. The push-pull formula implies

$$
\begin{aligned}
& \gamma_{*}\left(\left(e^{*}\right)^{2}\right)=\left(h^{*}\right)^{2} \gamma_{*}([X])=\left(h^{*}\right)^{2} \cdot d(d-1)^{2} h^{*}=d(d-1)^{2} *, \\
& j_{*}\left(\left(e^{*}\right)^{2}\right)=\alpha^{2} j_{*}\left(e^{2}\right)=\alpha^{2} h^{2} j_{*}([X])=\alpha^{2} h^{2} \cdot d h=\alpha^{2} d *,
\end{aligned}
$$

and thus $\alpha=d-1$. Hence, we have $(d-1) \gamma_{*}(e)=\gamma_{*}\left(e^{*}\right)=d(d-1)^{2}\left(h^{*}\right)^{2}$ and $\gamma_{*}(e)=$ $d(d-1)\left(h^{*}\right)^{2}$. Writing $[C]=\delta e$ for $\delta \in \mathbb{Z}$, we finally derive

$$
2 \operatorname{deg}\left(\mathcal{E}^{p}(X)\right)\left(h^{*}\right)^{2}=2\left[\mathcal{E}^{p}(X)\right]=\gamma_{*}([C])=\delta \gamma_{*}(e)=\delta d(d-1)\left(h^{*}\right)^{2}
$$

and $\delta=(d-2)\left(d^{3}-d^{2}+d-12\right)$ by Proposition 5.1. 
The numbers $a$ and $b$ of cusps and nodes of the curve $C$ are given by [25, Prop. 4.15]:

$$
\begin{aligned}
& a=4 d(d-2)(d-3)\left(d^{3}+3 d-16\right), \\
& b=\frac{1}{2} d(d-2)\left(d^{7}-4 d^{6}+7 d^{5}-45 d^{4}+114 d^{3}-111 d^{2}+548 d-960\right) .
\end{aligned}
$$

Since the curve $C$ does not have any other singularities, we can apply the intersection theoretic genus formula: the geometric genus of $C$ is given by the number of points in the 0-dimensional cycle $\frac{1}{2}\left([C]^{2}+K_{X}[C]\right)$ plus the number $1-a-b$ (cf. [12, Sec. 2.4.6]), where $K_{X}=(d-4) e$ is the canonical class (cf. [12, Sec. 1.4.3]):

$$
\begin{aligned}
j_{*}\left([C]^{2}+K_{X}[C]\right) & =\left(\delta^{2}+\delta(d-4)\right) j_{*}\left(e^{2}\right)=\left(\delta^{2}+\delta(d-4)\right) d * \quad \text { and } \\
\operatorname{genus}(C) & =\frac{1}{2}\left(\delta^{2}+\delta(d-4)\right) d+1-a-b \\
& =3 d^{6}-15 d^{5}+27 d^{4}-104 d^{3}+340 d^{2}-336 d+1 .
\end{aligned}
$$

The map $\gamma^{\prime}: C^{\prime} \rightarrow \mathcal{E}^{p}(X)^{\prime}$ between the normalizations of $C$ and $\mathcal{E}^{p}(X)$ is exactly ramified at the godrons, i.e., the points of tangency of the parabolic curve $P$ and the flecnodal curve $F$ on $X$ (see [28, pp. 229-231]). Thus, the number of those points is the number of points in the 0-dimensional cycle $\frac{1}{2}[P][F]=\frac{1}{2} \cdot 4(d-2) e \cdot(11 d-24) e$, which is $\frac{1}{2} j_{*}([P][F])=$ $2(d-2)(11 d-24) j_{*}\left(e^{2}\right)=2 d(d-2)(11 d-24) *$. We find the genus of $\mathcal{E}^{p}(X)$ by applying the Riemann-Hurwitz formula [16, Cor. 2.4] to $\gamma^{\prime}$ :

$$
2 \operatorname{genus}(C)-2=2\left(2 \operatorname{genus}\left(\mathcal{E}^{p}(X)\right)-2\right)+2 d(d-2)(11 d-24) .
$$

Hence, genus $\left(\mathcal{E}^{p}(X)\right)=\frac{1}{2}\left(3 d^{6}-15 d^{5}+27 d^{4}-115 d^{3}+386 d^{2}-384 d+2\right)$. Finally, the degree of the surface $\mathcal{E}(X)=\left(\mathcal{E}^{p}(X)\right)^{\vee}$ is the degree of the tangential surface of the curve $\mathcal{E}^{p}(X)$. The latter degree equals $2\left(\operatorname{deg}\left(\mathcal{E}^{p}(X)\right)+\operatorname{genus}\left(\mathcal{E}^{p}(X)\right)-1\right)-a=d(d-2)(d-3)\left(d^{2}+2 d-4\right)$ (see [27, Thm. 3.2]), since $a$ is also the number of cusps on $\mathcal{E}^{p}(X)$.

Proving the degrees of $\mathcal{C}(X)$ and $\mathcal{T}(X)$ is more technical. We will not include this here. One method is Colley's multiple point theory [8, 9]. Alternatively, one can write $\mathcal{C}(X)$ and $\mathcal{T}(X)$ as the intersection of loci of (principal) tangents in the fiber product $\Phi \times_{\operatorname{Gr}\left(1, \mathbb{P}^{3}\right)} \Phi$ or $\Phi \times_{\operatorname{Gr}\left(1, \mathbb{P}^{3}\right)} \Phi \times_{\operatorname{Gr}\left(1, \mathbb{P}^{3}\right)} \Phi$ and remove extra components in the intersection by blowing these up.

\section{Computing Visual Events}

Theorem 4.1 gives the degrees of the irreducible components of the visual event surface when $X$ is a general surface of degree $d$ in $\mathbb{P}^{3}$. Table 2 below summarizes these degrees for $d \leq 7$. One notices that the degrees are now much larger than those for curves in Table 1 .

The degrees in Table 2 pose a challenge because a homogeneous polynomial in four unknowns of degree $\delta$ can have as many as $\left(\begin{array}{c}\delta+3 \\ 3\end{array}\right)$ terms. For instance, if $X$ is a quintic surface then its flecnodal surface $\mathcal{F}(X)$ has degree $\delta=260$, so the expected number of terms is $\left(\begin{array}{c}\delta+3 \\ 3\end{array}\right)=2997411$. In this section we address this challenge. See Example 6.2 for a solution. 


\begin{tabular}{cccccc}
\hline$d$ & $\operatorname{deg}(\mathcal{F}(X))$ & $\operatorname{deg}(\mathcal{C}(X))$ & $\operatorname{deg}(\mathcal{T}(X))$ & $\operatorname{deg}(\mathcal{E}(X))$ & $\operatorname{deg}(\mathcal{P}(X))$ \\
\hline 3 & 0 & 0 & 0 & 0 & 30 \\
4 & 80 & 0 & 0 & 160 & 128 \\
5 & 260 & 510 & 0 & 930 & 330 \\
6 & 576 & 2448 & 624 & 3168 & 672 \\
7 & 1064 & 7308 & 3808 & 8260 & 1190 \\
\hline
\end{tabular}

Table 2: Degrees of the components of the visual event surface of a general surface

Throughout this section, we make use of the multiple root loci for binary forms. The ideals of these varieties are defined by homogeneous polynomials in the coefficients $c_{0}, c_{1}, \ldots c_{d}$ of

$$
c_{0} t^{d}+c_{1} t^{d-1}+c_{2} t^{d-2}+\cdots+c_{d-1} t+c_{d}
$$

For a partition $\lambda=\left(\lambda_{1}, \ldots, \lambda_{k}\right) \in \mathbb{N}^{k}$ with $\sum_{i=1}^{k} \lambda_{i} \leq d$, we write $\Delta_{\lambda}(d)$ for the homogeneous prime ideal in $\mathbb{R}\left[c_{0}, \ldots, c_{d}\right]$ whose variety consists of polynomials $(18)$ that have $k$ complex roots with multiplicities $\lambda_{1}, \ldots, \lambda_{k}$. The varieties are called multiple root loci in [23]. For example, $\Delta_{(4)}(d)$ is the prime ideal for polynomials of degree $d$ with one quadruple root.

Example 6.1. Let $d=4$ in $(18)$ and consider univariate quartics that have a single root of multiplicity four. These quartics are the points on a rational normal curve in $\mathbb{P}^{4}$. The prime ideal of this curve is $\Delta_{(4)}(4)$. It is generated by the six $2 \times 2$-minors of the $2 \times 4$-matrix

$$
\left(\begin{array}{cccc}
12 c_{0} & 3 c_{1} & 2 c_{2} & 3 c_{3} \\
3 c_{1} & 2 c_{2} & 3 c_{3} & 12 c_{4}
\end{array}\right) .
$$

The variety of quintics $(d=5)$ with one root of multiplicity four is the tangential surface of the rational normal curve in $\mathbb{P}^{5}$. Its ideal is the complete intersection of three quadrics:

$$
\Delta_{(4)}(5)=\left\langle 20 c_{0} c_{4}-8 c_{1} c_{3}+3 c_{2}^{2}, 50 c_{0} c_{5}-6 c_{1} c_{4}+c_{2} c_{3}, 20 c_{1} c_{5}-8 c_{2} c_{4}+3 c_{3}^{2}\right\rangle .
$$

Another multiple root locus was seen in Example 3.8. The ideal $\Delta_{(2,2)}(5)$ is minimally generated by 10 quintics. We used this to compute the edge surface of a degree 5 curve. $\diamond$

We refer to [23, Table 1] for details on the ideals $\Delta_{\lambda}(d)$. Some relevant instances are listed in Table 3. Its entries are copied from [23, Table 1]. For instance, the entry $6^{10}, 8^{38}$ in the last column means that $\Delta_{(3,2)}(7)$ is minimally generated by 10 sextics and 38 octics.

The ideals $\Delta_{\lambda}(d)$ can be computed either by direct implicitization, or by using subresultants [1]. The $i$-th subresultant $S_{i}\left(h_{1}, h_{2}\right)$ of two polynomials $h_{1}(t)$ and $h_{2}(t)$ is a polynomial of degree at most $i$ whose coefficients are the determinants of particular minors of the Sylvester matrix of $h_{1}$ and $h_{2}$. The vanishing of $S_{i}\left(h_{1}, h_{2}\right)$ for $0 \leq i \leq d-1$ means that the greatest common divisor (GCD) of $h_{1}$ and $h_{2}$ has degree at least $d$. Moreover, if $S_{d}\left(h_{1}, h_{2}\right)$ is not zero, it is exactly this GCD. If we let $h_{d}$ be the polynomial (18) and $h_{d}^{\prime}$ be its derivative with respect to $t$, then the condition that $h_{d}$ has roots with multiplicity $\lambda=\left(\lambda_{1}, \ldots, \lambda_{k}\right)$ is equivalent to the fact that the GCD of $h_{d}$ and $h_{d}^{\prime}$ has degree $\sum_{i=1}^{k}\left(\lambda_{i}-1\right)$ and has roots with multiplicities $\lambda^{\prime}=\left(\lambda_{1}-1, \ldots, \lambda_{k}-1\right)$. This allows us to compute the ideal $\Delta_{\lambda}(d)$ recursively. 


\begin{tabular}{cccccc}
\hline Ruled surface & Partition & $d=4$ & $d=5$ & $d=6$ & $d=7$ \\
\hline $\mathcal{F}(X)$ & $\lambda=(4)$ & $2^{6}$ & $2^{3}$ & $2^{1}, 3^{3}, 4^{1}$ & $4^{20}$ \\
$\mathcal{C}(X)$ & $\lambda=(3,2)$ & & $4^{28}$ & $4^{1}, 5^{3}, 6^{31}$ & $6^{10}, 8^{38}$ \\
$\mathcal{T}(X)$ & $\lambda=(2,2,2)$ & & & $4^{45}$ & $6^{78}$ \\
\hline
\end{tabular}

Table 3: The ideals $\Delta_{(\lambda)}(d)$ of multiple root loci relevant for visual events of surfaces

In what follows we assume that the ideals $\Delta_{\lambda}(d)$ have been pre-computed for $d \leq 7$. We use these data to compute the curves $\mathcal{F}^{\ell}(X), \mathcal{C}^{\ell}(X)$ and $\mathcal{T}^{\ell}(X)$ in the Grassmannian $\operatorname{Gr}\left(1, \mathbb{P}^{3}\right)$. The correspondence between the three multi-local events $\mathcal{F}, \mathcal{C}, \mathcal{T}$ and the three special partitions $\lambda$ was seen on the right side in Figure 2 , where $\lambda$ was denoted by $m$.

Let $f=f\left(x_{1}, x_{2}, x_{3}, x_{4}\right)$ be the polynomial of degree $d$ that defines the surface $X$. We parameterize the line in $\mathbb{P}^{3}$ with Plücker coordinates $q$ using a parameter $t$. For instance, we can write (2) dually as $z(t)=\left(q_{12}: t q_{12}: t q_{13}-q_{23}: t q_{14}-q_{24}\right)$. We substitute $z(t)$ into the polynomial $f$, and we regard $f(z(t))$ as a univariate polynomial in $t$, written as in (18). The coefficients $c_{i}$ are now homogeneous expressions of degree $d$ in the Plücker coordinates $q$. At this point, we substitute these expressions $c_{i}(q)$ into the generators of $\Delta_{\lambda}(d)$. The result is an ideal in the Plücker coordinates $q$ that defines the desired curve set-theoretically. The same method can be applied when local coordinates on the Grassmannian $\operatorname{Gr}\left(1, \mathbb{P}^{3}\right)$ are preferred. In this case, we parameterize the line in $\mathbb{P}^{3}$ by $z(t)=(1: t: \alpha+t \gamma: \beta+t \delta)$.

Example 6.2. Let $d=5$ and consider the smooth quintic surface $X$ defined by

$$
f=x_{1}^{5}+x_{2}^{5}+x_{3}^{5}+x_{4}^{5}+\left(x_{1}+x_{2}+x_{3}+x_{4}\right)^{5}+x_{1} x_{2} x_{3} x_{4}\left(x_{1}+x_{2}+x_{3}+x_{4}\right) .
$$

We compute the curve $\mathcal{F}^{\ell}(X)$ in $\operatorname{Gr}\left(1, \mathbb{P}^{3}\right)$ that represents the flecnodal surface. Its prime ideal has degree 260 and is generated by 10 sextics plus the Plücker quadric (1). This computation was done with the method above, starting from the ideal $\Delta_{(4)}(5)$ in $(19)$.

Let us shift gears and focus on the local events $\mathcal{P}$ and $\mathcal{E}$, seen on the left in Figure 2. We start with the parabolic surface $\mathcal{P}(X)$. Let $X$ be defined by a polynomial $f \in \mathbb{R}\left[x_{1}, x_{2}, x_{3}, x_{4}\right]$. The ideal $I(P)$ of the parabolic curve $P$ is defined by $f$ and the determinant of the Hessian matrix $H_{f}$. Consider the incidence variety of the parabolic curve and its tangent planes, that is $\left\{\left(x, T_{x}(X)\right) \mid x \in P\right\} \subset \mathbb{P}^{3} \times\left(\mathbb{P}^{3}\right)^{*}$. We compute the ideal of the incidence variety by adding the $2 \times 2$-minors of the matrix $\left(\begin{array}{ccc}\partial f / \partial x_{1} & \partial f / \partial x_{2} \\ y_{1} & \underset{y_{2}}{2} / \partial x_{3} & \partial f / \partial x_{4} \\ y_{3} & y_{4}\end{array}\right)$ to $I(P)$. We then saturate the resulting ideal by $\left\langle x_{1}, x_{2}, x_{3}, x_{4}\right\rangle$ and afterwards eliminate $x_{1}, x_{2}, x_{3}, x_{4}$. This furnishes the ideal of the dual curve $\mathcal{P}^{p}(X)$ in $\left(\mathbb{P}^{3}\right)^{*}$, which encodes the developable surface $\mathcal{P}(X)$.

Proposition 6.3. If $X$ is a general cubic surface, the curve $\mathcal{P}^{p}(X)$ is a complete intersection of a quartic and a sextic, obtained from the two basic invariants of ternary cubics.

Proof. A classical fact from invariant theory states that the ring of invariants for ternary cubics is generated by a quartic and a sextic, and these vanish precisely when the cubic has a cusp. We represent $X$ as the blow-up of $\mathbb{P}^{2}$ at six points, namely as the image of the map to $\mathbb{P}^{3}$ defined by four independent cubics $f_{1}, f_{2}, f_{3}, f_{4}$ in $x, y, z$ that vanish at these points. We 
now consider the cubic $y_{1} f_{1}+y_{2} f_{2}+y_{3} f_{3}+y_{4} f_{4}$, where $y_{1}, y_{2}, y_{3}, y_{4}$ are unknowns. Plugging this cubic into the two basic invariants gives the condition for a plane to meet $X$ in a cuspidal curve. Hence that locus in $\left(\mathbb{P}^{3}\right)^{*}$ is the complete intersection of a quartic and a sextic.

For a general parabolic point $x$ of $X$, the Hessian matrix $H_{f}(x)$ has rank three. Its kernel represents a unique point $p_{x}$ in $\mathbb{P}^{3}$. We use the following simple fact to compute $\mathcal{P}^{\ell}(X)$.

Lemma 6.4. For $x \in P$, the point $p_{x}$ lies on the unique principal tangent of $X$ at $x$.

Proof. The relation $x H_{f}(x) p_{x}^{T}=0$ holds. Euler's relation shows that $x H_{f}(x)$ is the gradient vector of $f$ at $x$. Hence $p_{x}$ lies on the tangent plane to $X$ at $x$. Furthermore, $p_{x}$ belongs to the principal tangent since $p_{x} H_{f}(x) p_{x}^{T}$ is zero. Hence $x$ and $p_{x}$ span the principal tangent.

The curve $\mathcal{P}^{\ell}(X) \subset \operatorname{Gr}\left(1, \mathbb{P}^{3}\right)$ is the collection of the lines spanned by a general parabolic point $x$ and the corresponding point $p_{x}$ from Lemma 6.4. This allows us to compute the ideal of $\mathcal{P}^{\ell}(X)$ in dual Plücker coordinates $q_{12}, q_{13}, \ldots, q_{34}$. First, we recover the ideal $I$ of the incidence variety $\left\{(x, y) \mid x \in P, y \in\right.$ ker $\left.H_{f}(x)\right\}$ by adding the four entries of the column vector $H_{f}(x) \cdot y$ to the ideal $I(P)=\left\langle f\right.$, det $\left.H_{f}(x)\right\rangle$. Secondly, we consider the map from the coordinate ring of the Grassmannian to the quotient ring of $I$ that maps Plücker coordinates $q_{i j}$ to the $2 \times 2$-minors of $\left(\begin{array}{llll}x_{1} & x_{2} & x_{3} & x_{4} \\ y_{1} & y_{2} & y_{3} & y_{4}\end{array}\right)$. The kernel of this ring map is the ideal of the curve $\mathcal{P}^{\ell}(X) \subset \operatorname{Gr}\left(1, \mathbb{P}^{3}\right)$. This ideal is generated by 4 cubics and 6 quartics, plus the Plücker quadric, in the unknowns $q_{12}, \ldots, q_{34}$. One verifies computationally that the ideal defines a curve of degree 30 in $\mathbb{P}^{5}$ for a cubic surface $X$. Of course, this curve is $\mathcal{P}^{\ell}(X)$.

Example 6.5. Let $d=3$ and consider the Fermat cubic $X$ defined by $f=x_{1}^{3}+x_{2}^{3}+x_{3}^{3}+x_{4}^{3}$. We can easily compute the ideal of the curve $\mathcal{P}^{\ell}(X)$ as described above, and from this we find the parabolic surface $\mathcal{P}(X)$. It decomposes into irreducible components of low degree:

$$
\begin{aligned}
&\left(x_{0}+x_{1}\right) \cdot\left(x_{0}+x_{2}\right) \cdot\left(x_{0}+x_{3}\right) \cdot\left(x_{1}+x_{2}\right) \cdot\left(x_{1}+x_{3}\right) \cdot\left(x_{2}+x_{3}\right) \\
& \cdot\left(x_{0}^{2}-x_{0} x_{1}+x_{1}^{2}\right) \cdot\left(x_{0}^{2}-x_{0} x_{2}+x_{2}^{2}\right) \cdot\left(x_{0}^{2}-x_{0} x_{3}+x_{3}^{2}\right) \\
& \cdot\left(x_{1}^{2}-x_{1} x_{2}+x_{2}^{2}\right) \cdot\left(x_{1}^{2}-x_{1} x_{3}+x_{3}^{2}\right) \cdot\left(x_{2}^{2}-x_{2} x_{3}+x_{3}^{2}\right) \\
& \cdot\left(x_{1}^{3}+x_{2}^{3}+x_{3}^{3}\right) \cdot\left(x_{0}^{3}+x_{2}^{3}+x_{3}^{3}\right) \cdot\left(x_{0}^{3}+x_{1}^{3}+x_{3}^{3}\right) \cdot\left(x_{0}^{3}+x_{1}^{3}+x_{2}^{3}\right) .
\end{aligned}
$$

This is one of the few cases where symbolic computation of the equation of $\mathcal{P}(X)$ is easy. $\diamond$

Example 6.6. Let $d=3$ and fix the cubic $f=x_{1}^{3}+x_{2}^{3}+x_{3}^{3}+x_{4}^{3}+\left(x_{1}+2 x_{2}+3 x_{3}+4 x_{4}\right)^{3}$. It defines our surface $X$. Using the method above, we rapidly compute the ideal of $\mathcal{P}^{\ell}(X)$. We demonstrate how to find the visual events of type $\mathcal{P}$ as the camera moves along a line.

Consider the line with parametric representation $z(t)=(t: 1: t-1: t+1)$ in $\mathbb{P}^{3}$. Let $Q$ be the skew-symmetric $4 \times 4$ matrix obtained from $(3)$ by substituting to dual Plücker coordinates. We add the four coordinates of $z(t) \cdot Q$ to the ideal of $\mathcal{P}^{\ell}$, we then saturate with respect to $\left\langle q_{12}, \ldots, q_{34}\right\rangle$, and thereafter we eliminate the unknowns $q_{i j}$. The result is

$$
\begin{gathered}
495403946635821355157683145728 t^{30}+4349505253226024309192581220352 t^{29} \\
+18437739306679654261938338946432 t^{28}+50562321054013553614808463278912 t^{27} \\
+\cdots \cdots-81509153943200707008 t^{2}-1885273424647073088 t-19650742648215232 .
\end{gathered}
$$


This polynomial has 30 distinct complex roots. Precisely 8 of them are real, namely

$$
\begin{aligned}
& \{-1.01358602985259876,-1.011352289518814,-0.600974923580648806,-0.35014676100811994, \\
& -0.2668550692437184,-0.191676056625314,-0.0811161566932513655,0.378943747730770221\} .
\end{aligned}
$$

These 8 roots mark the visual events of type $\mathcal{P}$ as the viewpoint travels along the line $z(t)$.

The univariate polynomial of degree 30 can also be computed from the dual curve $\mathcal{P}^{p}$. Let $g_{1}$ and $g_{2}$ be the polynomials in $y_{1}, y_{2}, y_{3}, y_{4}$ of degree four and six promised in Proposition 6.3. We augment the ideal $I\left(\mathcal{P}^{p}\right)=\left\langle g_{1}, g_{2}\right\rangle$ by the $3 \times 3$-minors of the $3 \times 4$-matrix

$$
\left(\begin{array}{cccc}
\partial g_{1} / \partial x_{1} & \partial g_{1} / \partial x_{2} & \partial g_{1} / \partial x_{3} & \partial g_{1} / \partial x_{4} \\
\partial g_{2} / \partial x_{1} & \partial g_{2} / \partial x_{2} & \partial g_{2} / \partial x_{3} & \partial g_{2} / \partial x_{4} \\
t & 1 & t-1 & t+1
\end{array}\right)
$$

We then saturate the resulting ideal by the ideal of the six $2 \times 2$-minors in first two rows, and finally we eliminate $x_{1}, x_{2}, x_{3}, x_{4}$. This gives the same polynomial of degree 30 in $t$.

We found that the computation of the edge surface $\mathcal{E}(X)$ is more challenging than that of the parabolic surface $\mathcal{P}(X)$. Consider the case when $X$ is a general quartic. Here, the surface $\mathcal{E}(X)$ has degree 160 , and hence so does the curve $\mathcal{E}^{\ell}(X)$ in $\operatorname{Gr}\left(1, \mathbb{P}^{3}\right)$. We succeeded in computing the ideal of this curve only for quartics $X$ that are singular or very special. For instance, if $X$ is the Fermat quartic then $\mathcal{E}(X)$ a surface of degree 80, with multiplicity 2. Since $\mathcal{E}(X)$ is developable, we could also try to use $\mathcal{E}^{p}(X)$ as an encoding. Unfortunately, the degree is then even higher. Namely, by Proposition 5.1, the dual curve $\mathcal{E}^{p}(X)$ has degree 480 in $\left(\mathbb{P}^{3}\right)^{*}$. The computation of edge surfaces $\mathcal{E}(X)$ definitely requires further research.

Acknowledgments. This project started in May 2016 at the GOAL workshop in Paris. We thank Mohab Safey El Din, Jean-Charles Faugère, Jon Hauenstein, and Jean Ponce for their help in the initial stages. We are also grateful to Joachim Rieger for an inspiring discussion on singularity theory, and to Emre Sertöz for helpful comments on intersection theory. Kathlén Kohn was funded by the Einstein Foundation Berlin. Bernd Sturmfels received partial support from the US National Science Foundation (DMS-1419018) and the Einstein Foundation Berlin. Matthew Trager was supported in part by the ERC advanced grant VideoWorld, the Institut Universitaire de France, the Inria-CMU associated team GAYA, and the ANR grant RECAP.

\section{References}

[1] J. Abdeljaoued, G.M. Diaz-Toca and L. Gonzalez-Vega: Minors of Bézout matrices, subresultants and the parametrization of the degree of the polynomial greatest common divisor, International Journal of Computer Mathematics 81 (2004) 1223-1238.

[2] V. I. Arnol'd: Singularities of smooth mappings, Russian Math. Surveys 23 (1968) 1-43.

[3] E. Arrondo, M. Bertolini and C. Turrini: A focus on focal surfaces, Asian Journal of Mathematics 5 (2001) 535-560. 
[4] M.C. Beltrametti, E. Carletti, G. Monti Bragadin and D. Gallarati: Lectures on Curves, Surfaces and Projective Varieties: a Classical View of Algebraic Geometry, EMS Textbooks in Mathematics, Vol. 9, European Mathematical Society, 2009.

[5] M-A. Bertin: On the singularities of the trisecant surface to a space curve, Matematiche (Catania) 53 (1998) 15-22.

[6] K. W. Bowyer and C. R. Dyer: Aspect graphs: An introduction and survey of recent results, International Journal of Imaging Systems and Technology 2 (1990) 315-328.

[7] A. Cayley: On the singularities of surfaces, The Cambridge and Dublin Mathematical Journal VII (1852) 166-171.

[8] S. Colley: Lines having specified contact with projective varieties, Proceedings of the 1984 Vancouver Conference in Algebraic Geometry 6 (1986) 47-70.

[9] S. Colley: Enumerating stationary multiple points, Advances in Math. 66 (1987) 149-170.

[10] I.V. Dolgachev: Classical Algebraic Geometry: a Modern View, Cambridge Univ. Press, 2012.

[11] W.L. Edge: The Theory of Ruled Surfaces, Cambridge University Press, 1931.

[12] D. Eisenbud and J. Harris: 3264 and All That: A Second Course in Algebraic Geometry, Cambridge University Press, 2016.

[13] D. Eisenbud and F-O. Schreyer: Resultants and Chow forms via exterior syzygies, Journal of the American Mathematical Society 16 (2003) 537-579.

[14] D.A. Forsyth and J. Ponce: Computer Vision: A Modern Approach, 2nd ed., Pearson, 2012.

[15] I.M. Gel'fand, M.M. Kapranov and A.V. Zelevinsky: Discriminants, Resultants and Multidimensional Determinants, Birkhäuser, Boston, 1994.

[16] R. Hartshorne: Algebraic Geometry, Springer-Verlag, New York and Heidelberg, 1977.

[17] P. Johansen: The geometry of the tangent developable, Computational methods for algebraic spline surfaces, Springer, Berlin, 2005, pp 95-106.

[18] Y. L. Kergosien: La famille des projections orthogonales d'une surface et ses singularités, Comptes Rendus Acad. Sc. Paris Sér.I Math 292 (1981) 929-932.

[19] J. J. Koenderink: Solid Shape, MIT Press, 1990.

[20] J. J. Koenderink and A. J. van Doorn: The singularities of the visual mapping, Biological Cybernetics 24 (1976) 51-59.

[21] K. Kohn: Coisotropic hypersurfaces in the Grassmannian, arXiv:1607.05932.

[22] K. Kohn, B.I.U. Nødland and P. Tripoli: Secants, bitangents, and their congruences, in Combinatorial Algebraic Geometry, Fields Institute, (eds. G. Smith and B. Sturmfels), to appear.

[23] H. Lee and B. Sturmfels: Duality of multiple root loci, Journal of Algebra 446 (2016) 499-526.

[24] S. Pae and J. Ponce: On computing structural changes in evolving surfaces and their appearance, International Journal of Computer Vision 43.2 (2001) 113-131.

[25] S. Petitjean: The complexity and enumerative geometry of aspect graphs of smooth surfaces, Algorithms in algebraic geometry and applications (Santander, 1994), 317-352, Progr. Math., 143, Birkhäuser, Basel, 1996.

[26] S. Petitjean, J. Ponce and D. Kriegman: Computing exact aspect graphs of curved objects: Algebraic Surfaces, International Journal of Computer Vision 9 (1992) 231-255. 
[27] R. Piene: Numerical characters of a curve in projective $n$-space, in Real and Complex Singularities, Oslo, (ed. P. Holm), 1976.

[28] R. Piene: Some formulas for a surface in $\mathbb{P}^{3}$, in Algebraic Geometry, (ed. L.D. Olson), Lecture Notes in Mathematics 687, Springer, Berlin, Heidelberg, 1978.

[29] R. Piene: Cuspidal projection of space curves, Mathematische Annalen 256 (1981) 95-119.

[30] O. A. Platonova: Projections of smooth surfaces, Journal of Mathematical Sciences 35.6 (1986) 2796-2808.

[31] J. Ponce and M. Hebert: On image contours of projective shapes, European Conference on Computer Vision. Springer International Publishing, 2014.

[32] J. Ponce and D. J. Kriegman: Computing exact aspect graphs of curved objects: Parametric surfaces, Department of Computer Science, University of Illinois at Urbana-Champaign, 1990.

[33] K. Ranestad and B. Sturmfels: On the convex hull of a space curve, Advances in Geometry 12 (2012) 157-178.

[34] J. H. Rieger: Global bifurcation sets and stable projections of nonsingular algebraic surfaces, International Journal of Computer Vision 7 (1992) 171-194.

[35] J.H. Rieger: Computing view graphs of algebraic surfaces, Journal of Symbolic Computation 16 (1993) 259-272.

[36] G. Salmon: A Treatise on the Analytic Geometry of Three Dimensions, 4th ed., Dublin, 1882.

[37] A. Seigal and B. Sturmfels: Real rank two geometry, Journal of Algebra 484 (2017) 310-333.

[38] B. Sturmfels: The Hurwitz form of a projective variety, Journal of Symbolic Computation 79 (2017) 186-196.

[39] R. Thom: Structural Stability and Morphogenesis, W.A. Benjamin, 1972.

\section{Authors' addresses:}

Kathlén Kohn, TU Berlin, Germany, kohn@math.tu-berlin.de.

Bernd Sturmfels, MPI Leipzig, Germany, and UC Berkeley, USA, bernd@mis .mpg . de, bernd@berkeley . edu.

Matthew Trager, Inria, École Normale Supérieure Paris, CNRS, PSL Research University, France, matthew.trager@inria.fr. 